\title{
On the generalized eigenvalue method for energies and matrix elements in lattice field theory
}

\section{\# $L P H A$ \\ Collaboration}

Benoit Blossier, ${ }^{a, b}$ Michele Della Morte, ${ }^{c, d}$ Georg von Hippel, ${ }^{a}$ Tereza Mendes $^{a, e}$ and Rainer Sommer ${ }^{a}$

${ }^{a}$ Deutsches Elektronen-Synchrotron DESY, Platanenallee 6, 15738 Zeuthen, Germany

${ }^{b}$ Laboratoire de Physique Théorique, Université Paris XI,

Bâtiment 210, F-91405 Orsay Cedex, France

${ }^{c}$ CERN, Physics Department, TH Division,

CH-1211 Geneva 23, Switzerland

${ }^{d}$ Institut für Kernphysik, University of Mainz,

Johann-Joachim-Becher-Weg 45, 55099 Mainz, Germany

${ }^{e}$ IFSC, University of São Paulo,

C.P. 369, CEP 13560-970, São Carlos SP, Brazil

E-mail: Benoit.Blossier@desy.de, morte@kph.uni-mainz.de,

Georg.von.Hippel@desy.de, mendes@ifsc.usp.br, Rainer.Sommer@desy.de

ABSTRACT: We discuss the generalized eigenvalue problem for computing energies and matrix elements in lattice gauge theory, including effective theories such as HQET. It is analyzed how the extracted effective energies and matrix elements converge when the time separations are made large. This suggests a particularly efficient application of the method for which we can prove that corrections vanish asymptotically as $\exp \left(-\left(E_{N+1}-E_{n}\right) t\right)$. The gap $E_{N+1}-E_{n}$ can be made large by increasing the number $N$ of interpolating fields in the correlation matrix.

We also show how excited state matrix elements can be extracted such that contaminations from all other states disappear exponentially in time.

As a demonstration we present numerical results for the extraction of ground state and excited B-meson masses and decay constants in static approximation and to order $1 / m_{\mathrm{b}}$ in HQET.

KeYwords: Lattice QCD, Lattice Quantum Field Theory, Lattice Gauge Field Theories, B-Physics

ArXiv ePrint: 0902.1265 


\section{Contents}

1 Introduction 1

2 The generalized eigenvalue problem 3

2.1 The basic idea 3

2.2 General statements about energies and matrix elements 4

3 Perturbation theory $\quad 6$

3.1 Application to the perturbations $C^{(1)} \quad 7$

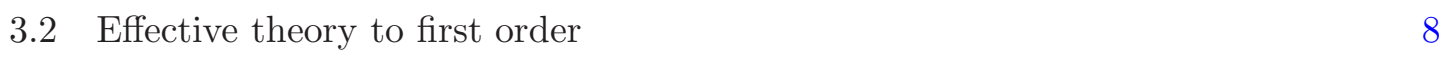

3.2.1 Energies 8

$\begin{array}{lll}3.2 .2 & \text { Matrix elements } & 9\end{array}$

4 Application to static-light $\mathrm{B}_{\mathrm{s}}$-mesons $\quad 10$

$\begin{array}{lll}5 & \text { Discussion } & 16\end{array}$

$\begin{array}{lr}\text { A Recursive perturbative expansion } & 18\end{array}$

$\begin{array}{lll}\text { A.1 The general case } & 18\end{array}$

$\begin{array}{lll}\text { A.2 The case of Euclidean QFT } & 19\end{array}$

$\begin{array}{lll}\text { A.3 Proof of eqs. (2.22)-(2.24) to all orders } & 19\end{array}$

\section{Introduction}

In the early days of lattice gauge theories, K. Wilson suggested to use a variational technique to compute energy levels in lattice gauge theory [1]. The general idea was quickly picked up and applied to the glueball spectrum [2,3] and to the static quark poten$\operatorname{tial}(\mathrm{s})[4,5]$.

The usual choice of a variational basis starts from some interpolating fields $O_{i}\left(x_{0}\right)$ already projected to a definite momentum and other quantum numbers such as parity. (Examples are given in the section on numerical results.) The associated Hilbert space operators $\hat{O}_{i}$ define states

$$
\left|\tilde{\phi}_{i}\right\rangle=\hat{O}_{i}|0\rangle \quad \text { and } \quad\left|\phi_{i}\right\rangle=\mathrm{e}^{-t_{0} \hat{H} / 2}\left|\tilde{\phi}_{i}\right\rangle,
$$

where $t_{0}$ is some time parameter and $\hat{H}$ the Hamilton operator. A variational principle is formulated by $\left(t>t_{0}\right)$

$$
\lambda_{1}\left(t, t_{0}\right)=\operatorname{Max}_{\left\{\alpha_{i}\right\}} \frac{\left\langle\phi\left|\mathrm{e}^{-\left(t-t_{0}\right) \hat{H}}\right| \phi\right\rangle}{\langle\phi \mid \phi\rangle}, \quad|\phi\rangle=\sum_{i=1}^{N} \alpha_{i}\left|\phi_{i}\right\rangle .
$$




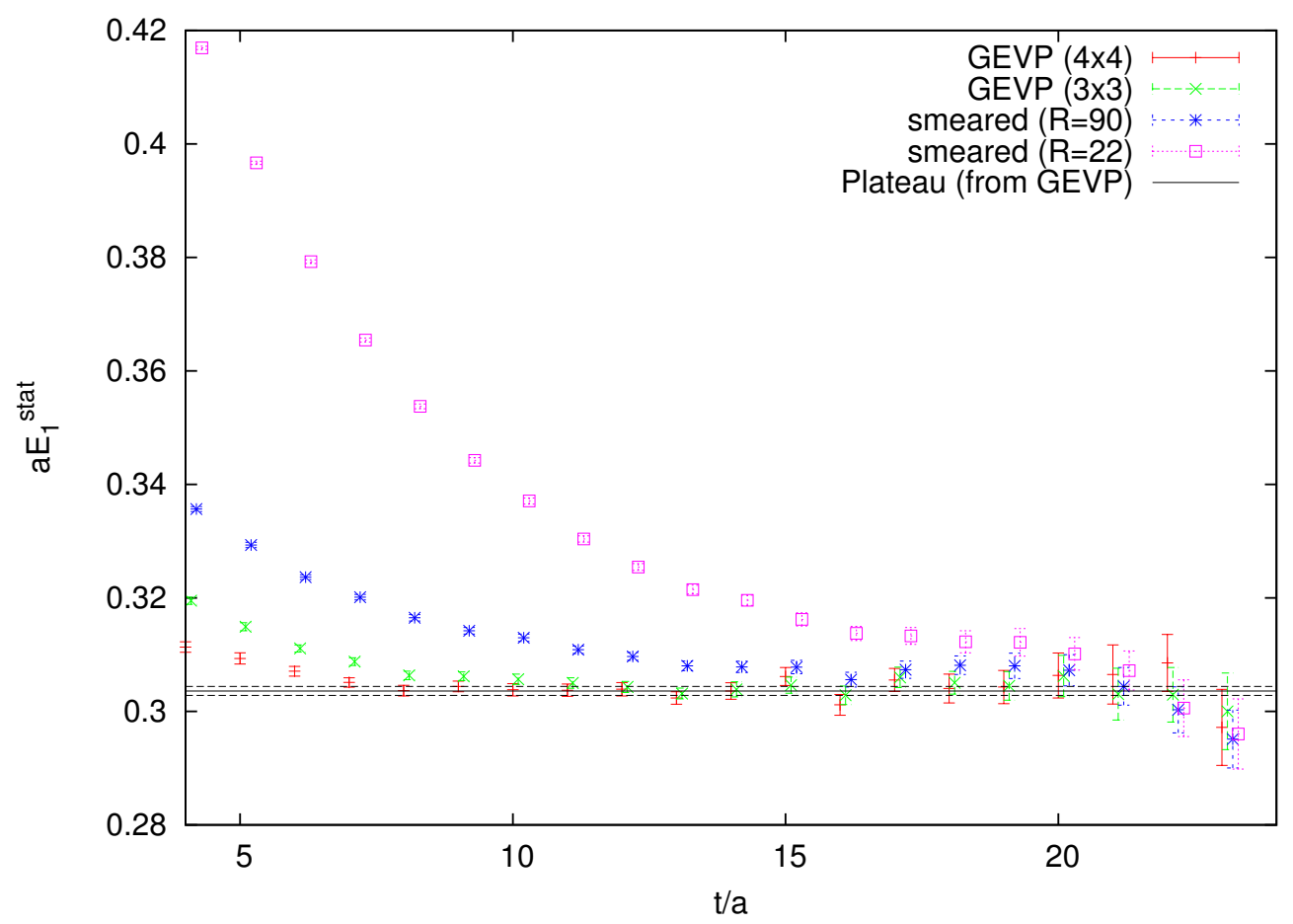

Figure 1. Plot of $a E_{1}^{\text {stat }}\left(t, t_{0}=4 a\right)$ against $t / a$ as obtained from the GEVP for a $3 \times 3$ and a $4 \times 4$ system, and from the effective mass plot of two correlators with different amounts of Gaussian smearing (22 and 90 iterations, respectively). The improved convergence of the GEVP solutions can be seen clearly.

Clearly $\lambda_{1}\left(t, t_{0}\right)$ yields a variational estimate of the lowest energy, $E_{1}$, with quantum numbers of $O_{i}$ via $\lambda_{1}\left(t, t_{0}\right) \approx \mathrm{e}^{-E_{1}\left(t-t_{0}\right)}$. At the same time $\lambda_{1}\left(t, t_{0}\right)$ is the largest eigenvalue of a generalized eigenvalue problem (GEVP), which we define below.

The GEVP is applicable beyond the computation of the ground state energy and it has been widely used in lattice field theories [6]. Recent examples are found in [7-19]. Corrections to the true energy levels decrease exponentially for large time $t$, when the energies are extracted from the generalized eigenvalues as described below [20].

Apart from the paper of Lüscher and Wolff [20], statements about corrections due to other energy levels seem to be absent in the literature. We here add such statements and suggest a special use of the GEVP, which we will show to be more efficient under certain conditions. We also treat the case of an effective theory and show numerical results for HQET. Furthermore, we show that the eigenvectors of the GEVP allow to construct operators (see eq. (2.16)) which applied to the vacuum yield states converging exponentially to eigenstates of the Hamiltonian.

With a proper condition for $t, t_{0}$ we prove that the convergence rate is $\sim \mathrm{e}^{-\left(E_{N+1}-E_{n}\right) t}$ for the energies and $\sim \mathrm{e}^{-\left(E_{N+1}-E_{n}\right) t_{0}}$ for matrix elements of the constructed states. The occurrence of the large gap $E_{N+1}-E_{n}$ is the main point of this paper. An earlier presentation of our work on this topic can be found in [21]. 
An indication of the effectiveness of the GEVP in suppressing contaminating contributions from excited states can be seen in figure 1, where we plot the effective energy of the ground state of a static-light pseudoscalar meson as obtained from different levels of Gaussian smearing and from the GEVP; for details, we refer to section 4.

\section{The generalized eigenvalue problem}

\subsection{The basic idea}

We start from a matrix of Euclidean space correlation functions

$$
\begin{aligned}
C_{i j}(t) & =\left\langle O_{i}(t) O_{j}^{*}(0)\right\rangle=\sum_{n=1}^{\infty} \mathrm{e}^{-E_{n} t} \psi_{n i} \psi_{n j}^{*}, & & i, j=1, \ldots, N \\
\psi_{n i} \equiv\left(\psi_{n}\right)_{i} & =\left\langle 0\left|\hat{O}_{i}\right| n\right\rangle & & E_{n}<E_{n+1} .
\end{aligned}
$$

Note that we have assumed non-degenerate energy levels. Space-time could be continuous, but in practice applications will be for discretized field theories. In that case, we assume that either the theory has a hermitian, positive transfer matrix as for the standard Wilson gauge theory $[22,23]$, or we consider $t, t_{0}$ large enough and the lattice spacing, $a$, small enough such that the correlation functions are well represented by a spectral representation and complex energy contributions are irrelevant [24, 25]. States $|n\rangle$ with $\langle m \mid n\rangle=\delta_{m n}$ are eigenstates of the Hamiltonian (logarithm of the transfer matrix) and all energies, $E_{n}$, have the vacuum energy subtracted. $O_{j}(t)$ are fields on a timeslice $t$ that correspond to Hilbert space operators $\hat{O}_{j}$ whose quantum numbers such as parity and flavor numbers are then also carried by the states $|n\rangle, n>0$. In the above formula, contributions due to a finite time extent of space-time are neglected, so the time extent $T$ has to be large. ${ }^{1}$

Before discussing the GEVP, let us comment briefly on a wider scope of applications. Besides the energy levels $E_{n}$ one may want to determine matrix elements $\langle 0|\hat{P}| n\rangle$ or $\langle m|\hat{P}| n\rangle$ of operators $\hat{P}$ that may or may not be in the set of operators $\left\{\hat{O}_{i}\right\}$. We will see that the GEVP provides a systematic approach to this problem as well.

The GEVP is defined by,

$$
C(t) v_{n}\left(t, t_{0}\right)=\lambda_{n}\left(t, t_{0}\right) C\left(t_{0}\right) v_{n}\left(t, t_{0}\right), \quad n=1, \ldots, N, \quad t>t_{0} .
$$

Lüscher and Wolff showed that one can use it to determine systematically also excited states, namely [20]

$$
\begin{aligned}
E_{n} & =\lim _{t \rightarrow \infty} E_{n}^{\mathrm{eff}}\left(t, t_{0}\right), \\
E_{n}^{\mathrm{eff}}\left(t, t_{0}\right) & =-\partial_{t} \log \lambda_{n}\left(t, t_{0}\right) \equiv-\frac{1}{a}\left[\log \lambda_{n}\left(t+a, t_{0}\right)-\log \lambda_{n}\left(t, t_{0}\right)\right],
\end{aligned}
$$

where $a$ is the lattice spacing.

\footnotetext{
${ }^{1}$ See [26] for a discussion of finite $T$ when the $O_{i}$ carry vacuum quantum numbers.
} 
For a while (until section 2.2) we now assume a simplified case, which helps to understand the usefulness of $E_{n}^{\mathrm{eff}}$ and $v_{n}$. The simplification is that only $N$ states contribute,

$$
C_{i j}(t)=C_{i j}^{(0)}(t)=\sum_{n=1}^{N} \mathrm{e}^{-E_{n} t} \psi_{n i} \psi_{n j}^{*} .
$$

We introduce the dual (time-independent) vectors $u_{n}$, defined by

$$
\left(u_{n}, \psi_{m}\right)=\delta_{m n}, \quad m, n \leq N
$$

with $\left(u_{n}, \psi_{m}\right) \equiv \sum_{i=1}^{N}\left(u_{n}^{*}\right)_{i} \psi_{m i}$. Inserting into eq. (2.5) gives

$$
C^{(0)}(t) u_{n}=\mathrm{e}^{-E_{n} t} \psi_{n}, \quad C^{(0)}(t) u_{n}=\lambda_{n}^{(0)}\left(t, t_{0}\right) C^{(0)}\left(t_{0}\right) u_{n}
$$

So the GEVP is solved by

$$
\lambda_{n}^{(0)}\left(t, t_{0}\right)=\mathrm{e}^{-E_{n}\left(t-t_{0}\right)}, \quad v_{n}\left(t, t_{0}\right) \propto u_{n}
$$

and there is an orthogonality for all $t$ of the form

$$
\left(u_{m}, C^{(0)}(t) u_{n}\right)=\delta_{m n} \rho_{n}(t), \quad \rho_{n}(t)=\mathrm{e}^{-E_{n} t} .
$$

These equations mean that the hermitean conjugates of the operators

$$
\hat{\mathcal{A}}_{n}=\sum_{i=1}^{N}\left(u_{n}^{*}\right)_{i} \hat{O}_{i} \equiv\left(u_{n}, \hat{O}\right),
$$

create the eigenstates of the Hamilton operator,

$$
|n\rangle=\hat{\mathcal{A}}_{n}^{\dagger}|0\rangle, \quad \hat{H}|n\rangle=E_{n}|n\rangle .
$$

Consequently matrix elements are easily obtained. For example

$$
\langle 0|\hat{P}| n\rangle=\left\langle 0\left|\hat{P} \hat{\mathcal{A}}_{n}^{\dagger}\right| 0\right\rangle,
$$

translates into correlation functions of $P, O_{i}$.

\subsection{General statements about energies and matrix elements}

Let us now come back to the general case eq. (2.1). The idea is to solve the GEVP, eq. (2.2), "at large time" where the contribution of states $n>N$ is small. We then consider the effective energies, eq. (2.4), and effective operators ${ }^{2}$

$$
\hat{\mathcal{A}}_{n}^{\mathrm{eff}}\left(t, t_{0}\right)=\mathrm{e}^{-\hat{H} t} \hat{\mathcal{Q}}_{n}^{\mathrm{eff}}\left(t, t_{0}\right),
$$

\footnotetext{
${ }^{2}$ For simplicity we discuss a special case of a more general class of operators depending on several times, which then all have to be taken large. An example is

$$
\begin{aligned}
\hat{\mathcal{Q}}_{n}^{\mathrm{eff}} & =R_{n}\left(\hat{O}, v_{n}\left(t_{1}, t_{0}\right)\right) \\
R_{n} & =\left(v_{n}\left(t_{1}, t_{0}\right), C\left(t_{2}\right) v_{n}\left(t_{1}, t_{0}\right)\right)^{-1 / 2} \frac{\lambda_{n}\left(t_{0}+t_{2} / 2, t_{0}\right)}{\lambda_{n}\left(t_{0}+t_{2}, t_{0}\right)} .
\end{aligned}
$$




$$
\begin{aligned}
\hat{\mathcal{Q}}_{n}^{\mathrm{eff}}\left(t, t_{0}\right) & =R_{n}\left(\hat{O}, v_{n}\left(t, t_{0}\right)\right), \\
R_{n} & =\left(v_{n}\left(t, t_{0}\right), C(t) v_{n}\left(t, t_{0}\right)\right)^{-1 / 2} \frac{\lambda_{n}\left(t_{0}+t / 2, t_{0}\right)}{\lambda_{n}\left(t_{0}+t, t_{0}\right)} .
\end{aligned}
$$

Corrections to the large time asymptotics are parameterized by

$$
\begin{aligned}
E_{n}^{\mathrm{eff}}\left(t, t_{0}\right) & =E_{n}+\varepsilon_{n}\left(t, t_{0}\right), \\
\mathrm{e}^{-\hat{H} t}\left(\hat{\mathcal{Q}}_{n}^{\mathrm{eff}}\left(t, t_{0}\right)\right)^{\dagger}|0\rangle & =|n\rangle+\sum_{n^{\prime}=1}^{\infty} \pi_{n n^{\prime}}\left(t, t_{0}\right)\left|n^{\prime}\right\rangle .
\end{aligned}
$$

The terms $\varepsilon_{n}, \pi_{n n^{\prime}}$ will disappear exponentially at large times. Note that in the literature the energy levels are sometimes not extracted like that and rather the standard effective masses of correlators made from $\tilde{\mathcal{A}}_{n}=\left(O, v_{n}\left(t_{1}, t_{0}\right)\right)$ are used with fixed $t_{1}, t_{0}$ and the question of the size of the corrections is left open. However, the form eq. (2.18) has a theoretical advantage as it was shown in [20] that (at fixed $t_{0}$ )

$$
\varepsilon_{n}\left(t, t_{0}\right)=\mathrm{O}\left(\mathrm{e}^{-\Delta E_{n} t}\right), \quad \Delta E_{n}=\min _{m \neq n}\left|E_{m}-E_{n}\right| .
$$

This is non-trivial as it allows to obtain the excited levels with corrections which vanish in the limit of large $t$, keeping $t_{0}$ fixed. However, it appears from this formula that the corrections can be large when there is an energy level close to the desired one. This is the case in interesting phenomena such as string breaking [27-31], where in numerical applications the corrections appeared to be very small despite the formula above. Also in static-light systems the gaps are typically only around $\Delta E_{n} \approx 400 \mathrm{MeV}$, and in full QCD with light quarks, a small gap $\Delta E_{n} \approx 2 m_{\pi}$ appears.

Our contribution to the issue is a more complete discussion of the correction $\varepsilon_{n}$ to $E_{n}$ as well as the definition of the effective operator, eq. (2.16), and a discussion of the corrections $\pi_{n n^{\prime}}$ to its matrix elements. It turns out that in all this, a very useful case is to consider the situation

$$
t_{0} \geq t / 2
$$

e.g. with $t-t_{0}=$ const. or $2 \geq t / t_{0}=$ const. and take $t_{0}$ (in practice moderately) large. In this region of $t, t_{0}$ the second order perturbations in higher states $n>N$ are not larger than the first order ones. We can then show that

$$
\begin{aligned}
\varepsilon_{n}\left(t, t_{0}\right) & =\mathrm{O}\left(\mathrm{e}^{-\Delta E_{N+1, n} t}\right), \quad \Delta E_{m, n}=E_{m}-E_{n}, \\
\pi_{n n^{\prime}}\left(t, t_{0}\right) & =\mathrm{O}\left(\mathrm{e}^{-\Delta E_{N+1, n} t_{0}}\right), \quad \text { at fixed } t-t_{0} \\
\pi_{1 n}\left(t, t_{0}\right) & =\mathrm{O}\left(\mathrm{e}^{-\Delta E_{N+1,1} t_{0}} \mathrm{e}^{-\Delta E_{2,1}\left(t-t_{0}\right)}\right)+\mathrm{O}\left(\mathrm{e}^{-\Delta E_{N+1,1} t}\right) .
\end{aligned}
$$

The large gaps $\Delta E_{N+1, n}$ can solve the problem of close-by levels for example in the stringbreaking situation, but also speed up the general convergence very much. For example in static-light systems $\Delta E_{6,1} \approx 2 \mathrm{GeV}$ translates into a gain of a factor of 5 in time separation.

Eq. (2.23) means that matrix elements of (time-)local operators $\hat{P}$ and the associated field $P$ can be computed via

$$
\left\langle 0\left|\hat{\mathcal{Q}}_{n}^{\mathrm{eff}} \mathrm{e}^{-\hat{H} t} \hat{P} \mathrm{e}^{-\hat{H} t}\left(\hat{\mathcal{Q}}_{n^{\prime}}^{\mathrm{eff}}\right)^{\dagger}\right| 0\right\rangle=\left\langle\mathcal{Q}_{n}^{\mathrm{eff}}(2 t) P(t)\left(\mathcal{Q}_{n^{\prime}}^{\mathrm{eff}}(0)\right)^{*}\right\rangle=\left\langle n|\hat{P}| n^{\prime}\right\rangle+\mathrm{O}\left(\mathrm{e}^{-\Delta E_{N+1, n} t_{0}}\right)
$$


and similarly for $\langle n|\hat{P}| 0\rangle$.

We now turn to an outline of the proof of these statements, delegating the technical part to an appendix.

\section{Perturbation theory}

We start from the solutions above for $C=C^{(0)}$ and treat the higher states as perturbations. This perturbative evaluation was already set up by F. Niedermayer and P. Weisz a while ago [32] but never published. We noted the rôle of $t_{0} \geq t / 2$, the form of the corrections to the effective operators defined above and could show that these relations hold to all orders in the perturbative expansion.

We want to obtain $\lambda_{n}$ and $v_{n}$ in a perturbation theory in $\epsilon$, where

$$
A v_{n}=\lambda_{n} B v_{n}, \quad A=A^{(0)}+\epsilon A^{(1)}, \quad B=B^{(0)}+\epsilon B^{(1)} .
$$

All these $N \times N$ matrices are assumed to be hermitian, $B$ and $B^{(0)}$ are assumed to be positive. We will set

$$
\begin{array}{ll}
A^{(0)}=C^{(0)}(t), & \epsilon A^{(1)}=C^{(1)}(t), \\
B^{(0)}=C^{(0)}\left(t_{0}\right), & \epsilon B^{(1)}=C^{(1)}\left(t_{0}\right)
\end{array}
$$

in the end with

$$
C_{i j}^{(1)}(t)=\sum_{n=N+1}^{\infty} \mathrm{e}^{-E_{n} t} \psi_{n i} \psi_{n j}^{*},
$$

which is hermitian and positive under the assumptions listed in the previous section. A possible choice for $\epsilon$ is $\epsilon=\mathrm{e}^{-\left(E_{N+1}-E_{N}\right) t_{0}}$ which becomes arbitrarily small for large $t_{0}$. The solutions of the lowest-order equation

$$
A^{(0)} v_{n}^{(0)}=\lambda_{n}^{(0)} B^{(0)} v_{n}^{(0)},
$$

satisfy an orthogonality relation

$$
\left(v_{n}^{(0)}, B^{(0)} v_{m}^{(0)}\right)=\rho_{n} \delta_{n m}
$$

as in eq. (2.9) above. Non-degenerate eigenvalues $\lambda_{n}^{(0)}>\lambda_{n+1}^{(0)}$ are assumed. Expanding

$$
\lambda_{n}=\lambda_{n}^{(0)}+\epsilon \lambda_{n}^{(1)}+\epsilon^{2} \lambda_{n}^{(2)} \ldots, \quad v_{n}=v_{n}^{(0)}+\epsilon v_{n}^{(1)}+\epsilon^{2} v_{n}^{(2)} \ldots
$$

with the orthogonality condition

$$
\left(v_{n}^{(k)}, B^{(0)} v_{n}^{(0)}\right)=0, \quad k=1,2, \ldots,
$$

we get for the first two orders

$$
A^{(0)} v_{n}^{(1)}+A^{(1)} v_{n}^{(0)}=\lambda_{n}^{(0)}\left[B^{(0)} v_{n}^{(1)}+B^{(1)} v_{n}^{(0)}\right]+\lambda_{n}^{(1)} B^{(0)} v_{n}^{(0)},
$$




$$
\begin{aligned}
A^{(0)} v_{n}^{(2)}+A^{(1)} v_{n}^{(1)}= & \lambda_{n}^{(0)}\left[B^{(0)} v_{n}^{(2)}+B^{(1)} v_{n}^{(1)}\right]+\lambda_{n}^{(1)}\left[B^{(0)} v_{n}^{(1)}+B^{(1)} v_{n}^{(0)}\right] \\
& +\lambda_{n}^{(2)} B^{(0)} v_{n}^{(0)} .
\end{aligned}
$$

With the orthogonality, eq. (3.6), of the lowest order vectors, one obtains just like in ordinary quantum mechanics perturbation theory the solutions for eigenvalues and the eigenvectors

$$
\begin{aligned}
& \lambda_{n}^{(1)}=\rho_{n}^{-1}\left(v_{n}^{(0)}, \Delta_{n} v_{n}^{(0)}\right), \quad \Delta_{n} \equiv A^{(1)}-\lambda_{n}^{(0)} B^{(1)} \\
& v_{n}^{(1)}=\sum_{m \neq n} \alpha_{n m}^{(1)} v_{m}^{(0)}, \quad \alpha_{n m}^{(1)}=\rho_{m}^{-1} \frac{\left(v_{m}^{(0)}, \Delta_{n} v_{n}^{(0)}\right)}{\lambda_{n}^{(0)}-\lambda_{m}^{(0)}} \\
& \lambda_{n}^{(2)}=\sum_{m \neq n} \rho_{n}^{-1} \rho_{m}^{-1} \frac{\left|\left(v_{m}^{(0)}, \Delta_{n} v_{n}^{(0)}\right)\right|^{2}}{\lambda_{n}^{(0)}-\lambda_{m}^{(0)}}-\rho_{n}^{-2}\left(v_{n}^{(0)}, \Delta_{n} v_{n}^{(0)}\right)\left(v_{n}^{(0)}, B^{(1)} v_{n}^{(0)}\right) .
\end{aligned}
$$

A recursion formula for the higher-order coefficients is given in appendix A.

We note that for our case of interest, $t_{0}$ can be chosen large enough such that the perturbation theory is absolutely convergent. It therefore suffices to discuss the large $t_{0}, t$ asymptotics order by order in the expansion in order to show the detailed form of corrections, in particular that they are controlled by the large gap $\Delta E_{N+1, n}$. Convergence of the expansion is guaranteed for the following reason. As in quantum mechanics, the perturbation theory can be set up with the help of the resolvent depending on a complex variable $z$. The positions of its poles yield the (here generalized) eigenvalues. The resolvent for the GEVP is

$$
G(z)=(z B-A)^{-1}=G_{0}(z) \sum_{k=0}^{\infty} \epsilon^{k}\left[\left(A^{(1)}-z B^{(1)}\right) G_{0}(z)\right]^{k}
$$

in terms of the unperturbed resolvent $G_{0}(z)=\left(z B^{(0)}-A^{(0)}\right)^{-1}$. The series expansion eq. (3.14) converges for large $t_{0}$ (and $t>t_{0}$ ) since $\left\|\epsilon\left(z B^{(1)}-A^{(1)}\right)\right\|<\left\|G_{0}^{-1}(z)\right\|$ holds at sufficiently large $t_{0}$ (note that the resolvent is needed only away from the zeros of $G_{0}^{-1}(z)$ ).

\subsection{Application to the perturbations $C^{(1)}$}

Now we insert our specific problem eq. (3.2), eq. (3.3). With a representation (for $m>n$ )

$$
\begin{aligned}
\left(\lambda_{n}^{(0)}-\lambda_{m}^{(0)}\right)^{-1} & =\left(\lambda_{n}^{(0)}\right)^{-1}\left(1-\mathrm{e}^{-\left(E_{m}-E_{n}\right)\left(t-t_{0}\right)}\right)^{-1} \\
& =\left(\lambda_{n}^{(0)}\right)^{-1} \sum_{l=0}^{\infty} \mathrm{e}^{-l\left(E_{m}-E_{n}\right)\left(t-t_{0}\right)},
\end{aligned}
$$

and some algebra spelled out in appendix A.2 one can show that eqs. (2.22)-(2.24) hold at any order in $\epsilon$.

In particular, we list below the explicit expressions up to second order in $\epsilon$, keeping only leading terms in the sums. They demonstrate how the condition $t_{0} \geq t / 2$ comes about.

For the effective energies we have ( $a$ is the lattice spacing, cf. eq. (2.4))

$$
\varepsilon_{n}\left(t, t_{0}\right) \sim \mathrm{e}^{-\left(E_{N+1}-E_{n}\right) t}\left[1-\mathrm{e}^{-\left(E_{N+1}-E_{n}\right) a}\right] c_{n, n, N+1}
$$




$$
\begin{aligned}
& +\sum_{m>n} \mathrm{e}^{-\left(2 E_{N+1}-E_{n}-E_{m}\right) t_{0}} \mathrm{e}^{-\left(E_{m}-E_{n}\right)\left(t-t_{0}\right)}\left[1-\mathrm{e}^{-\left(E_{m}-E_{n}\right) a}\right]\left|c_{n, m, N+1}\right|^{2} \\
& -\sum_{m<n} \mathrm{e}^{-\left(2 E_{N+1}-E_{n}-E_{m}\right) t_{0}} \mathrm{e}^{-\left(E_{n}-E_{m}\right)\left(t-t_{0}\right)}\left[1-\mathrm{e}^{-\left(E_{n}-E_{m}\right) a}\right]\left|c_{n, m, N+1}\right|^{2}
\end{aligned}
$$

where the first term (line) comes from order $\epsilon$ in the perturbative expansion and the second and third terms come from order $\epsilon^{2}$. Also, we have defined

$$
c_{n, m, l}=\left(u_{n}, \psi_{l}\right)\left(\psi_{l}, u_{m}\right)
$$

We see that, due to cancellations of $t$-independent terms in the effective energy, the firstorder correction is independent of $t_{0}$ and exponentially suppressed in $t$ with the large energy gap $E_{N+1}-E_{n}$ as coefficient. (This correction is positive, while the sign of the combined second-order one depends on the energy shifts for the states around $E_{n}$.) At fixed $t_{0}$ and asymptotically large $t$ the second-order corrections dominate because they follow the slower decay eq. (2.20). However, simple inspection of the above corrections shows that the $t_{0^{-}}$ dependent prefactors supress the second order correction sufficiently when $t_{0} \geq t / 2$. Then the first-order one dominates, confirming eq. (2.22).

For the amplitudes $\pi_{n n^{\prime}}\left(t, t_{0}\right)$ we have main contributions at first order in $\epsilon$ for $n^{\prime} \neq n$, $n^{\prime} \leq N$. These contributions from $n^{\prime}>n$ and $n^{\prime}<n$ are given respectively by

$$
\pi_{n n^{\prime}}\left(t, t_{0}\right)=-c_{n, n^{\prime}, N+1} \mathrm{e}^{-\left(E_{N+1}-E_{n}\right) t_{0}} \mathrm{e}^{-\left(E_{n^{\prime}}-E_{n}\right)\left(t-t_{0}\right)}
$$

and

$$
\pi_{n n^{\prime}}\left(t, t_{0}\right)=c_{n, n^{\prime}, N+1} \mathrm{e}^{-\left(E_{N+1}-E_{n}\right) t_{0}} .
$$

Note that in the $n^{\prime}>n$ case the value $n^{\prime}=n+1$ has a leading exponential behavior, whereas for $n^{\prime}<n$ all values of $n^{\prime}$ have the same exponential behavior.

\subsection{Effective theory to first order}

In an effective theory such as HQET, all correlation functions

$$
C_{i j}(t)=C_{i j}^{\mathrm{stat}}(t)+\omega C_{i j}^{1 / \mathrm{m}}(t)+\mathrm{O}\left(\omega^{2}\right)
$$

are computed in an expansion in a small parameter, $\omega$, which we here consider to first order only. The notation is taken from HQET where $\omega \propto 1 / m_{\mathrm{b}}$. It also helps to avoid a confusion with the perturbation theory in terms of $C^{(1)}$ treated before.

\subsubsection{Energies}

We start our analysis from the GEVP in the full theory, eq. (2.2), and use the form of the correction terms of the effective energies $\left(t \leq 2 t_{0}\right)$

$$
E_{n}^{\mathrm{eff}}\left(t, t_{0}\right)=a^{-1} \log \frac{\lambda_{n}\left(t, t_{0}\right)}{\lambda_{n}\left(t+a, t_{0}\right)}=E_{n}+\mathrm{O}\left(\mathrm{e}^{-\Delta E_{N+1, n} t}\right),
$$


see the discussion above. Expanding this equation in $\omega$, we arrive at

$$
\begin{aligned}
E_{n}^{\mathrm{eff}}\left(t, t_{0}\right) & =E_{n}^{\mathrm{eff}, \mathrm{stat}}\left(t, t_{0}\right)+\omega E_{n}^{\mathrm{eff}, 1 / \mathrm{m}}\left(t, t_{0}\right)+\mathrm{O}\left(\omega^{2}\right) \\
E_{n}^{\mathrm{eff}, \text { stat }}\left(t, t_{0}\right) & =a^{-1} \log \frac{\lambda_{n}^{\text {stat }}\left(t, t_{0}\right)}{\lambda_{n}^{\text {stat }}\left(t+a, t_{0}\right)} \\
E_{n}^{\mathrm{eff}, 1 / \mathrm{m}}\left(t, t_{0}\right) & =\frac{\lambda_{n}^{1 / \mathrm{m}}\left(t, t_{0}\right)}{\lambda_{n}^{\text {stat }}\left(t, t_{0}\right)}-\frac{\lambda_{n}^{1 / \mathrm{m}}\left(t+a, t_{0}\right)}{\lambda_{n}^{\text {stat }}\left(t+a, t_{0}\right)}
\end{aligned}
$$

with the behavior at large time,

$$
\begin{aligned}
& E_{n}^{\mathrm{eff}, \text { stat }}\left(t, t_{0}\right)=E_{n}^{\mathrm{stat}}+\beta_{n}^{\mathrm{stat}} \mathrm{e}^{-\Delta E_{N+1, n}^{\mathrm{stat}} t}+\cdots, \\
& E_{n}^{\mathrm{eff}, 1 / \mathrm{m}}\left(t, t_{0}\right)=E_{n}^{1 / \mathrm{m}}+\left[\beta_{n}^{1 / \mathrm{m}}-\beta_{n}^{\mathrm{stat}} t \Delta E_{N+1, n}^{1 / \mathrm{m}}\right] \mathrm{e}^{-\Delta E_{N+1, n}^{\text {stat }} t}+\cdots .
\end{aligned}
$$

Following the beginning of section 3 we now take the lowest order correlator to define the unperturbed GEVP,

$$
C^{\text {stat }}(t) v_{n}^{\text {stat }}\left(t, t_{0}\right)=\lambda_{n}^{\text {stat }}\left(t, t_{0}\right) C^{\text {stat }}\left(t_{0}\right) v_{n}^{\text {stat }}\left(t, t_{0}\right),
$$

whose eigenvectors with normalization $\left(v_{m}^{\text {stat }}\left(t, t_{0}\right), C^{\text {stat }}\left(t_{0}\right) v_{n}^{\text {stat }}\left(t, t_{0}\right)\right)=\delta_{m n}$ are then needed in the formula

$$
\frac{\lambda_{n}^{1 / \mathrm{m}}\left(t, t_{0}\right)}{\lambda_{n}^{\text {stat }}\left(t, t_{0}\right)}=\left(v_{n}^{\text {stat }}\left(t, t_{0}\right),\left[\left[\lambda_{n}^{\text {stat }}\left(t, t_{0}\right)\right]^{-1} C^{1 / \mathrm{m}}(t)-C^{1 / \mathrm{m}}\left(t_{0}\right)\right] v_{n}^{\text {stat }}\left(t, t_{0}\right)\right)
$$

for the first-order corrections in $\omega$. We note that the finite time corrections in eq. (3.24) comprise of both a term $\mathrm{e}^{-\Delta E_{N+1, n}^{\text {stat }} t}$ and one with an extra factor of $t$, however only their prefactors are new, once $\Delta E_{N+1, n}^{\text {stat }}$ is known from an analysis of the lowest order eigenvalues. Due to the large gap $\Delta E_{N+1, n}^{\text {stat }}$, the corrections disappear quickly with time.

\subsubsection{Matrix elements}

The discussion of matrix elements starts from the operators $\hat{\mathcal{Q}}_{n}^{\text {eff }}$, eq. (2.16). Their expansion

$$
\hat{\mathcal{Q}}_{n}^{\text {eff }}=R_{n}^{\text {stat }}\left(v_{n}^{\text {stat }}\left(t, t_{0}\right), \hat{O}\right)+\omega\left[R_{n}^{1 / \mathrm{m}}\left(v_{n}^{\text {stat }}\left(t, t_{0}\right), \hat{O}\right)+R_{n}^{\text {stat }}\left(v_{n}^{1 / \mathrm{m}}\left(t, t_{0}\right), \hat{O}\right)\right]
$$

is given in terms of

$$
\begin{aligned}
R_{n}^{\text {stat }}= & \left(v_{n}^{\text {stat }}\left(t, t_{0}\right), C^{\text {stat }}(t) v_{n}^{\text {stat }}\left(t, t_{0}\right)\right)^{-1 / 2} \frac{\lambda_{n}^{\text {stat }}\left(t_{0}+t / 2, t_{0}\right)}{\lambda_{n}^{\text {stat }}\left(t_{0}+t, t_{0}\right)}, \\
\frac{R_{n}^{1 / \mathrm{m}}}{R_{n}^{\text {stat }}}= & -\frac{1}{2} \frac{\left(v_{n}^{\text {stat }}\left(t, t_{0}\right), C^{1 / \mathrm{m}}(t) v_{n}^{\text {stat }}\left(t, t_{0}\right)\right)}{\left(v_{n}^{\text {stat }}\left(t, t_{0}\right), C^{\text {stat }}(t) v_{n}^{\text {stat }}\left(t, t_{0}\right)\right)} \\
& +\frac{\lambda_{n}^{1 / \mathrm{m}}\left(t_{0}+t / 2, t_{0}\right)}{\lambda_{n}^{\text {stat }}\left(t_{0}+t / 2, t_{0}\right)}-\frac{\lambda_{n}^{1 / \mathrm{m}}\left(t_{0}+t, t_{0}\right)}{\lambda_{n}^{\text {stat }}\left(t_{0}+t, t_{0}\right)}
\end{aligned}
$$

and the first order perturbation to $v_{n}$,

$$
v_{n}^{1 / \mathrm{m}}=\sum_{k \neq n=1}^{N} v_{k}^{\text {stat }} \frac{\left(v_{k}^{\text {stat }},\left[C^{1 / \mathrm{m}}(t)-\lambda_{n}^{\text {stat }}\left(t, t_{0}\right) C^{1 / \mathrm{m}}\left(t_{0}\right)\right] v_{n}^{\text {stat }}\right)}{\lambda_{n}^{\text {stat }}\left(t, t_{0}\right)-\lambda_{k}^{\text {stat }}\left(t, t_{0}\right)} .
$$


A simple matrix element is then

$$
p_{n}^{\text {eff }}\left(t, t_{0}\right)=\left\langle 0\left|\hat{\mathcal{Q}}_{n}^{\text {eff }} \mathrm{e}^{-\hat{H} t} \hat{P}\right| 0\right\rangle=R_{n}\left(v_{n}\left(t, t_{0}\right), C_{\mathrm{P}}\right), \quad\left(C_{\mathrm{P}}\right)_{j}=\left\langle O_{j}(0) P(t)\right\rangle .
$$

For example when $P$ denotes the time component of an axial current, it yields the decay constant of the associated ground state meson via,

$$
p_{1}=\langle 0|\hat{P}| 1\rangle,
$$

with the $\omega$-expansion

$$
\begin{aligned}
p_{n}^{\mathrm{eff}}\left(t, t_{0}\right) & =p_{n}^{\mathrm{eff}, \mathrm{stat}}\left(t, t_{0}\right)\left(1+\omega p_{n}^{\mathrm{eff}, 1 / \mathrm{m}}\left(t, t_{0}\right)+\mathrm{O}\left(\omega^{2}\right)\right) \\
p_{n}^{\mathrm{eff}, 1 / \mathrm{m}}\left(t, t_{0}\right) & =\frac{R_{n}^{1 / \mathrm{m}}}{R_{n}^{\text {stat }}}+\frac{\left(v_{n}^{\text {stat }}\left(t, t_{0}\right), C_{\mathrm{P}}^{1 / \mathrm{m}}(t)\right)}{\left(v_{n}^{\text {stat }}\left(t, t_{0}\right), C_{\mathrm{P}}^{\text {stat }}(t)\right)}+\frac{\left(v_{n}^{1 / \mathrm{m}}\left(t, t_{0}\right), C_{\mathrm{P}}^{\text {stat }}(t)\right)}{\left(v_{n}^{\text {stat }}\left(t, t_{0}\right), C_{\mathrm{P}}^{\text {stat }}(t)\right)} .
\end{aligned}
$$

Specializing further, the large time asymptotics of the effective ground state matrix element has the following form

$$
\begin{aligned}
p_{1}^{\text {eff }}\left(t, t_{0}\right) & \sim p_{1}+\gamma \mathrm{e}^{-\Delta E_{N+1,1} t_{0}}, \\
p_{1}^{\text {eff, stat }}\left(t, t_{0}\right) & \sim p_{1}^{\text {stat }}+\gamma^{\text {stat }} \mathrm{e}^{-\Delta E_{N+1,1}^{\text {stat }} t_{0}}, \\
p_{1}^{\text {eff }, 1 / \mathrm{m}}\left(t, t_{0}\right) & \sim p_{1}^{1 / \mathrm{m}}+\mathrm{e}^{-\Delta E_{N+1,1}^{\text {stat }} t_{0}}\left[\gamma^{1 / \mathrm{m}}-\gamma^{\text {stat }} p_{1}^{1 / \mathrm{m}}-t_{0} \Delta E_{N+1,1}^{1 / \mathrm{m}} \gamma^{\text {stat }}\right] .
\end{aligned}
$$

We note that the correction term proportional to $t_{0} \mathrm{e}^{-\Delta E_{N+1,1}^{\text {stat }} t_{0}}$ is entirely fixed by a first order analysis of the energies and the lowest order one of the matrix element.

\section{Application to static-light $\mathrm{B}_{\mathrm{s}}-$ mesons}

We have carried out numerical tests of eqs. (3.23)-(3.39) in the context of static-light $B_{s}$ mesons in quenched HQET with a HYP2 static quark [33-35] and a non-perturbatively $\mathrm{O}(a)$-improved [36, 37] Wilson valence (strange) quark. ${ }^{3}$ Our lattices are $L^{3} \times T$ with $L \approx$ $1.5 \mathrm{fm}, T=2 L$ and periodic boundary conditions in all directions. For this demonstration we have chosen $a=0.07 \mathrm{fm}\left(\beta=6.2885, \kappa_{s}=0.1349798\right)$ and use an ensemble of 100 quenched configurations. A more detailed analysis including multiple lattice spacings to enable us to take the continuum limit is in progress.

Strange quark propagators are computed using a variant of the Dublin method [38]. We use approximate instead of exact low modes and employ even-odd preconditioning in order to reduce both the size of the eigenvalue problem to be solved for the low modes and the number of noise sources to be used for the stochastic estimator by a factor of 2 ; this leads to a significant reduction in effort and is expected to also reduce the amount of noise introduced by the stochastic estimation of the short-distance contributions [39].

\footnotetext{
${ }^{3}$ One may object that a quenched calculation does not satisfy our basic premise, eq. (2.1). However the quark considered here is rather heavy and experience has shown that quenching represents a small modification of the full theory in such cases. Clearly a demonstration in a unitary theory will be welcome.
} 
The interpolating fields are constructed using quark bilinears

$$
\begin{aligned}
O_{k}(x) & =\bar{\psi}_{\mathrm{h}}(x) \gamma_{0} \gamma_{5} \psi_{1}^{(k)}(x) \\
O_{k}^{*}(x) & =\bar{\psi}_{1}^{(k)}(x) \gamma_{0} \gamma_{5} \psi_{\mathrm{h}}(x)
\end{aligned}
$$

of identical Dirac structure, but with different levels of Gaussian smearing [40] for the light quark fields

$$
\psi_{1}^{(k)}(x)=\left(1+\kappa_{G} \Delta\right)^{R_{k}} \psi_{\mathrm{l}}(x),
$$

where the gauge fields in the covariant Laplacian are first smeared with 3 iterations of (spatial) APE smearing [41, 42]. We use $R_{k}=22,45,67,90,135,180,225$ with $\kappa_{G}=0.1$. The local $\left(R_{0}=0\right)$ operator is also included in order to be able to compute the decay constant. Here and throughout, $\psi_{\mathrm{h}}(x)$ denotes the static quark field.

For these operators, we compute the following correlators:

$$
\begin{aligned}
C_{i j}^{\mathrm{stat}}(t) & =\frac{a^{13}}{L^{6} T} \sum_{\vec{x}, \vec{y}, t_{x}}\left\langle O_{i}\left(\vec{y}, t+t_{x}\right) O_{j}^{*}\left(\vec{x}, t_{x}\right)\right\rangle, \\
C_{i}^{\delta A}(t) & =\frac{a^{14}}{L^{6} T} \sum_{\vec{x}, \vec{y}, t_{x}}\left\langle O_{i}\left(\vec{y}, t+t_{x}\right) O_{\delta A}^{*}\left(\vec{x}, t_{x}\right)\right\rangle, \\
C_{i j}^{\mathrm{kin}}(t) & =\frac{a^{21}}{L^{9} T} \sum_{\vec{x}, \vec{y}, \vec{z}, t_{x}, t_{x} \leq t_{y} \leq t_{x}+t}\left\langle O_{i}\left(\vec{z}, t+t_{x}\right) O_{\operatorname{kin}}\left(\vec{y}, t_{y}\right) O_{j}^{*}\left(\vec{x}, t_{x}\right)\right\rangle, \\
C_{i j}^{\mathrm{spin}}(t) & =\frac{a^{21}}{L^{9} T} \sum_{\vec{x}, \vec{y}, \vec{z}, t_{x}, t_{x} \leq t_{y} \leq t_{x}+t}\left\langle O_{i}\left(\vec{z}, t+t_{x}\right) O_{\operatorname{spin}}\left(\vec{y}, t_{y}\right) O_{j}^{*}\left(\vec{x}, t_{x}\right)\right\rangle,
\end{aligned}
$$

where

$$
\begin{aligned}
O_{\delta A}(\vec{x}, t) & =\bar{\psi}_{\mathrm{h}}(\vec{x}, t) \gamma_{0} \gamma_{5}\left[\vec{\gamma} \cdot \vec{D} \psi_{1}^{(0)}\right](\vec{x}, t), \\
O_{\text {kin }}(\vec{x}, t) & =\bar{\psi}_{\mathrm{h}}(\vec{x}, t)\left[\vec{D}^{2} \psi_{\mathrm{h}}\right](\vec{x}, t), \\
O_{\text {spin }}(\vec{x}, t) & =\bar{\psi}_{\mathrm{h}}(\vec{x}, t)\left[\vec{\sigma} \cdot \vec{B} \psi_{\mathrm{h}}\right](\vec{x}, t) .
\end{aligned}
$$

The correlator $C^{\delta A}$ is introduced to ensure the axial local current is correct up to both $\mathcal{O}(a)$ and $\mathcal{O}\left(1 / m_{\mathrm{b}}\right)$, whereas the last two correlators are the $\mathcal{O}\left(1 / m_{\mathrm{b}}\right)$ terms from the HQET action.

The energy of a state $|n\rangle$ is given by

$$
E_{n}=E_{n}^{\mathrm{stat}}+\omega_{\mathrm{kin}} E_{n}^{\mathrm{kin}}+\omega_{\mathrm{spin}} E_{n}^{\mathrm{spin}}+\delta m
$$

and its decay constant factorizes as

$$
f_{B}^{(n)} \sqrt{M_{B}^{(n)} / 2}=Z_{A}^{\mathrm{HQET}} p_{n}^{\mathrm{stat}}\left(1+\omega_{\mathrm{kin}} p_{n}^{\mathrm{kin}}+\omega_{\mathrm{spin}} p_{n}^{\mathrm{spin}}+c_{A}^{\mathrm{HQET}} p_{n}^{\delta A}\right)
$$

where $Z_{A}^{\mathrm{HQET}}, \omega_{\text {kin }}, \omega_{\text {spin }}, c_{A}^{\mathrm{HQET}}$ and $\delta m$ are (divergent) matching constants between the QCD action and currents and their HQET counterpart. A strategy to compute them has been presented in $[43,44]$ and we quote their approximate numerical values [39] at 
$\beta=6.2885\left(Z_{A}^{\mathrm{HQET}} \sim 1, \omega_{\text {kin }} / a \sim 0.4, \omega_{\text {spin }} / a \sim 0.7, c_{A}^{\mathrm{HQET}} / a \sim-0.6\right)$ only as an illustration of the expected size of their contributions.

The resulting $8 \times 8$ correlator matrices $C(t)$ are symmetrized and then truncated to $N \times N$ matrices $C^{(N \times N)}(t)$ by projecting with the $N$ eigenvectors belonging to the $N$ largest eigenvalues of $C^{\text {stat }}\left(t_{i}\right)$ :

$$
\begin{aligned}
& C^{\text {stat }}\left(t_{i}\right) b_{n}=\lambda_{n} b_{n} \\
& C_{n m}^{(N \times N)}(t)=b_{n}^{\dagger} C(t) b_{m}, n, m \leq N .
\end{aligned}
$$

For $N$ not too large, this helps to avoid numerical instabilities in the GEVP that could otherwise lead to large errors [45]. We use $t_{i}=2 a$.

For each of the resulting $N \times N$ correlators, we solve the static GEVP and compute the static and $\mathrm{O}\left(1 / m_{\mathrm{b}}\right)$ energies and matrix elements as per eqs. (3.23)-(3.24) and (3.30)-(3.36). This gives a series of estimates $E_{n}^{N \text {,stat }}\left(t, t_{0}\right), p_{n}^{N \text {,stat }}\left(t, t_{0}\right)$ etc. with associated statistical errors, which we determine by a full Jackknife analysis.

To arrive at final numbers for $E_{n}, p_{n}$, we first need to estimate the size of the systematic errors coming from the higher excited states. To do this, we perform a fit of the form

$$
E_{n}^{N, \text { stat }}\left(t, t_{0}\right)=E_{n}^{\text {stat }}+\beta_{n, N}^{\text {stat }} \mathrm{e}^{-\left(E_{N+1}^{\text {stat }}-E_{n}^{\text {stat }}\right) t}
$$

(cf. eq. (3.25)) to the GEVP results for $E_{n}^{N \text {,stat }}\left(t, t_{0}\right)$, fitting the data at $N=3, \ldots, 5$, $t_{0} / a=3, \ldots, 6$ and $n=1, \ldots, 6$ simultaneously. Then, using the values of $E_{n}^{\text {stat }}$ and $\beta_{n, N}^{\text {stat }}$ determined from this fit as (fixed) input parameters, we fit $E_{n}^{N, \text {,in }}\left(t, t_{0}\right)$ and $E_{n}^{N, \text { spin }}\left(t, t_{0}\right)$ by

$$
\begin{aligned}
& E_{n}^{N, \text { kin }}\left(t, t_{0}\right)=E_{n}^{\mathrm{kin}}+\left[\beta_{n, N}^{\mathrm{kin}}-\beta_{n, N}^{\mathrm{stat}} t\left(E_{N+1}^{\mathrm{kin}}-E_{n}^{\mathrm{kin}}\right)\right] \mathrm{e}^{-\left(E_{N+1}^{\mathrm{stat}}-E_{n}^{\text {stat }}\right) t} \\
& E_{n}^{N, \text { spin }}\left(t, t_{0}\right)=E_{n}^{\text {spin }}+\left[\beta_{n, N}^{\text {spin }}-\beta_{n, N}^{\text {stat }} t\left(E_{N+1}^{\text {spin }}-E_{n}^{\text {spin }}\right)\right] \mathrm{e}^{-\left(E_{N+1}^{\text {stat }}-E_{n}^{\text {stat }}\right) t}
\end{aligned}
$$

(cf. eq. (3.26)) in the same manner. Subsequently, we also fit $p_{n}^{N \text {,stat }}\left(t, t_{0}\right)$ to

$$
p_{n}^{N \text {,stat }}\left(t, t_{0}\right)=p_{n}^{\text {stat }}+\gamma_{n, N}^{\text {stat }} \mathrm{e}^{-\left(E_{N+1}^{\text {stat }}-E_{n}^{\text {stat }}\right) t_{0}}
$$

(cf. eq. (3.38)), and $p_{n}^{N, \text {,kin }}\left(t, t_{0}\right), p_{n}^{N \text {,spin }}\left(t, t_{0}\right)$ and $p_{n}^{N, \delta A}\left(t, t_{0}\right)$ to

$$
\begin{aligned}
& p_{n}^{N, \text { kin }}\left(t, t_{0}\right)=p_{n}^{\mathrm{kin}}+\left[\gamma_{n, N}^{\mathrm{kin}}-\gamma_{n, N}^{\mathrm{stat}} p_{n}^{\mathrm{kin}}-\gamma_{n, N}^{\mathrm{stat}} t_{0}\left(E_{N+1}^{\mathrm{kin}}-E_{n}^{\mathrm{kin}}\right)\right] \mathrm{e}^{-\left(E_{N+1}^{\mathrm{stat}}-E_{n}^{\text {stat }}\right) t} \\
& p_{n}^{N, \text { spin }}\left(t, t_{0}\right)=p_{n}^{\text {spin }}+\left[\gamma_{n, N}^{\mathrm{spin}}-\gamma_{n, N}^{\text {stat }} p_{n}^{\text {spin }}-\gamma_{n, N}^{\text {stat }} t_{0}\left(E_{N+1}^{\mathrm{spin}}-E_{n}^{\mathrm{spin}}\right)\right] \mathrm{e}^{-\left(E_{N+1}^{\mathrm{stat}}-E_{n}^{\text {stat }}\right) t} \\
& p_{n}^{N, \delta A}\left(t, t_{0}\right)=p_{n}^{\delta A}+\left[\gamma_{n, N}^{\delta A}-\gamma_{n, N}^{\text {stat }} p_{n}^{\delta A}\right] \mathrm{e}^{-\left(E_{N+1}^{\text {stat }}-E_{n}^{\text {stat }}\right) t}
\end{aligned}
$$

(cf. eq. (3.39)), using the previously determined values for $E_{n}^{\text {stat }}, \gamma_{n, N}^{\text {stat }}, E_{n}^{\text {kin }}$ and $E_{n}^{\text {spin }}$ as fixed input parameters.

Since our fits do not include contributions beyond the leading excited state correction, the errors estimated from the fits may not be very reliable. In particular we consider the fitted values of $E_{4}^{\text {stat }}, \ldots, E_{6}^{\text {stat }}$ as rough estimates only, which may have significant additional systematic errors. We therefore use the fitted values only for the purpose of 


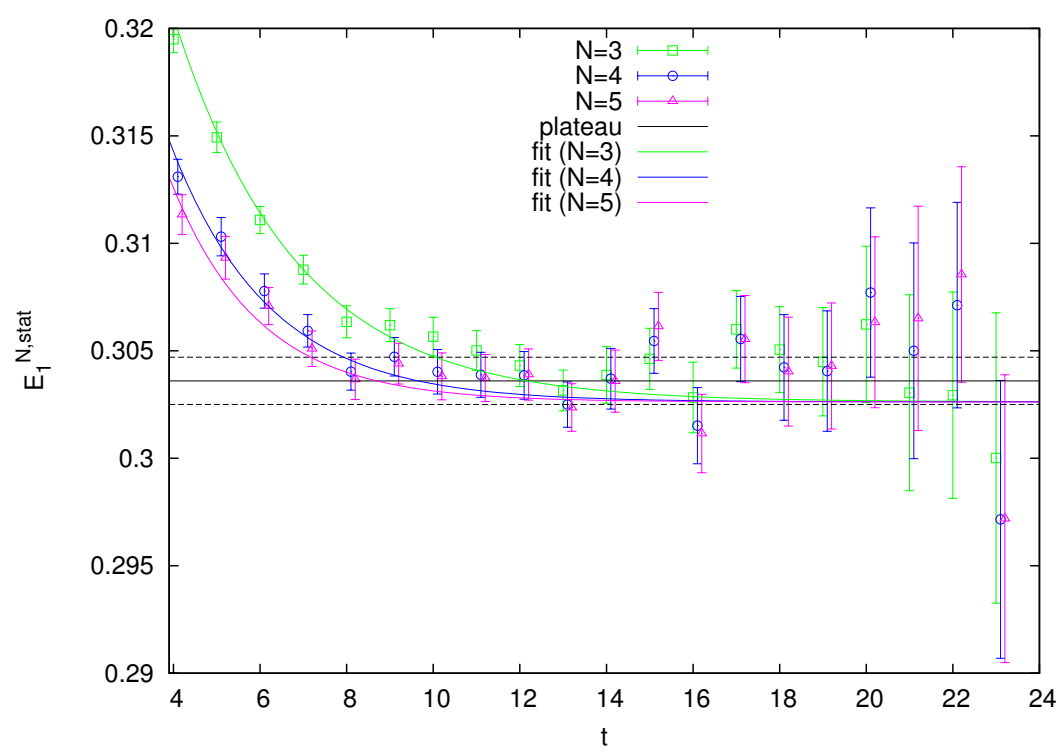

Figure 2. Plot of $E_{1}^{N \text {,stat }}\left(t, t_{0}=4 a\right)$ against $t$. All quantities are given in lattice units. Data points are displaced horizontally for better visibility. Also shown for the purposes of comparison are the fit curves from eq. (3.25) and the plateau given in table 1. Note that the curves are fitted to the total data set, not just the points shown in the plot, and that the plateau is obtained at a different $t_{0}$ than shown here.

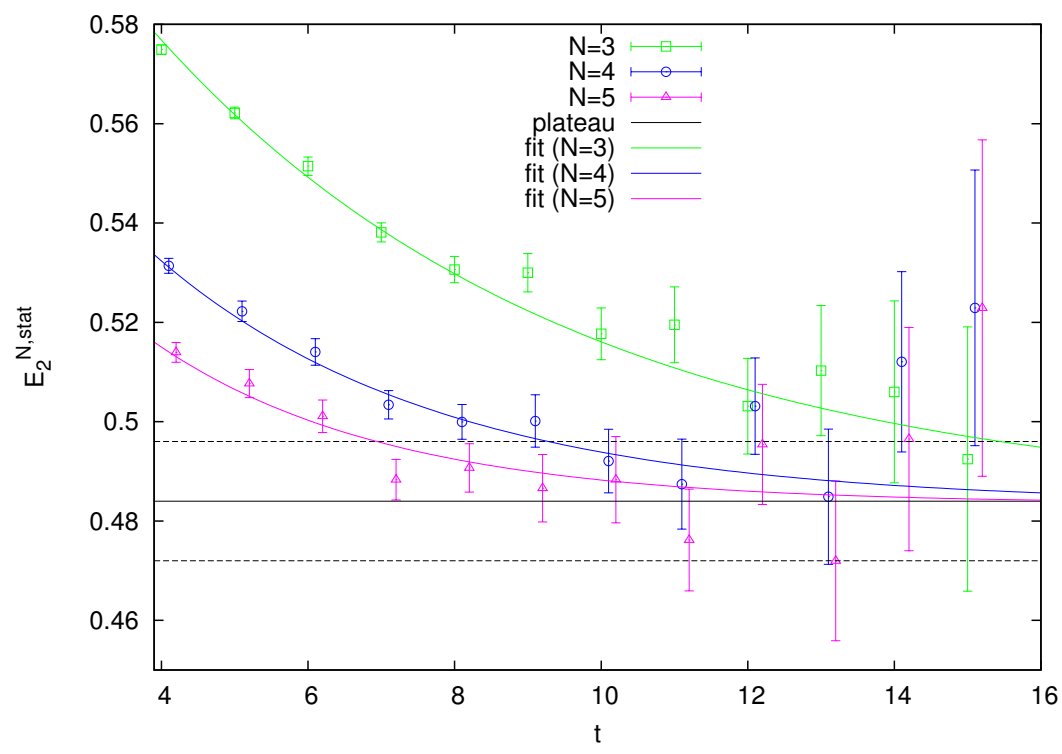

Figure 3. Plot of $E_{2}^{N \text {,stat }}\left(t, t_{0}=4 a\right)$ against $t$ in the same style as figure 2 .

determining the systematic correction $\epsilon(t)$ coming from the excited states. The good quality of the fit that can be seen from figures 2 to 9 makes them very suitable for that purpose. In particular we note that we find the $E_{n}^{N}\left(t, t_{0}\right)$ to be essentially independent of $t_{0}$, as predicted by our fit formulas. 


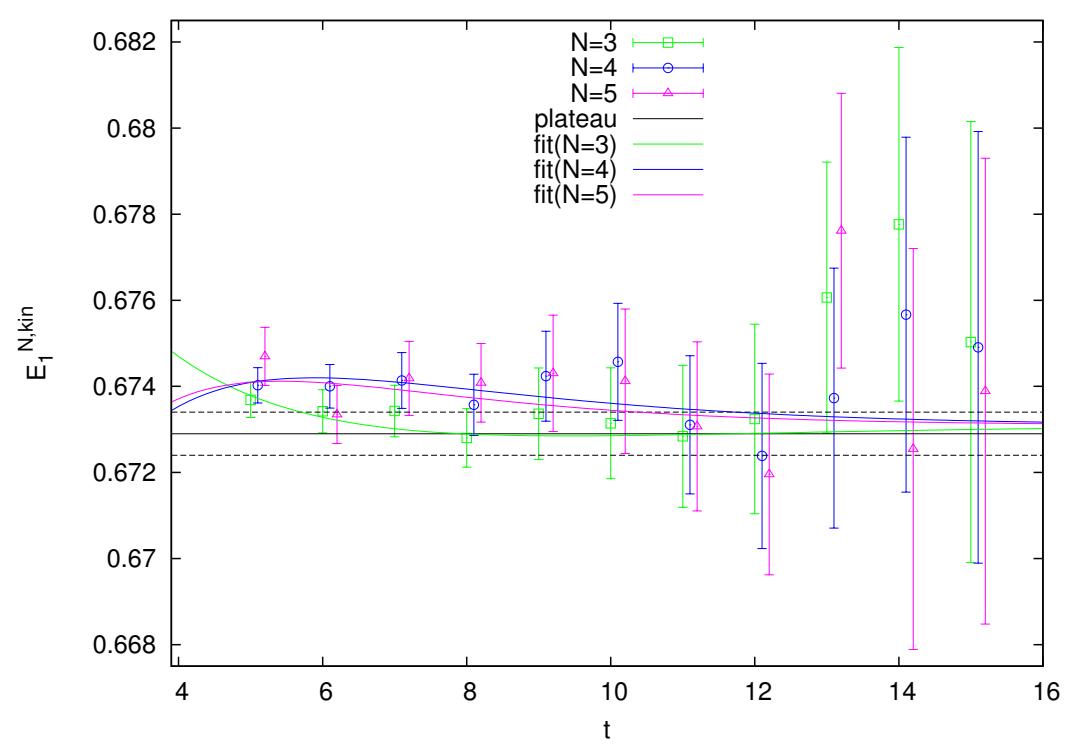

Figure 4. Plot of $E_{1}^{N, \text { kin }}\left(t, t_{0}=4 a\right)$ against $t$ in the same style as figure 2 .

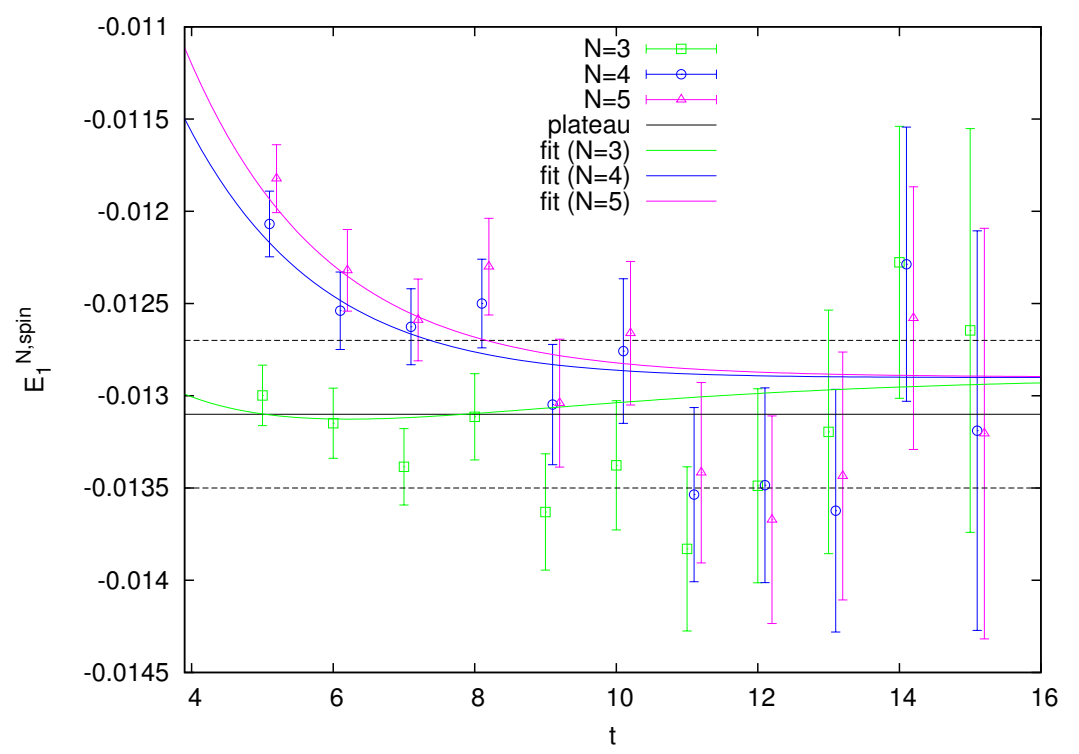

Figure 5. Plot of $E_{1}^{N \text {,spin }}\left(t, t_{0}=4 a\right)$ against $t$ in the same style as figure 2 .

For a reliable estimate of our quantities of interest, we calculate plateau averages of the energies from $t=t_{\min } \geq t_{0}$ to $t=2 t_{0}$ at each $N$ and $t_{0}$ (and from $t_{0}=t_{0, \min } \geq t / 2$ to $t_{0}=t$ at each $N$ and $t$ for the matrix elements). As our final estimate we take that plateau average for which the absolute sum $\sigma_{\text {tot }}=\sigma_{\text {stat }}+\sigma_{\text {sys }}$ of the statistical error $\sigma_{\text {stat }}$ of the plateau average and the maximum systematic error $\sigma_{\mathrm{sys}}=\epsilon\left(t_{\min }\right)$ becomes minimal, subject to the constraint that $\sigma_{\text {sys }}<\sigma_{\text {stat }}$. We impose the latter constraint in order to ensure that the systematic errors are subdominant.

Figures 2 to 9 show plots of our results at different values of $N$ along with the fits and 


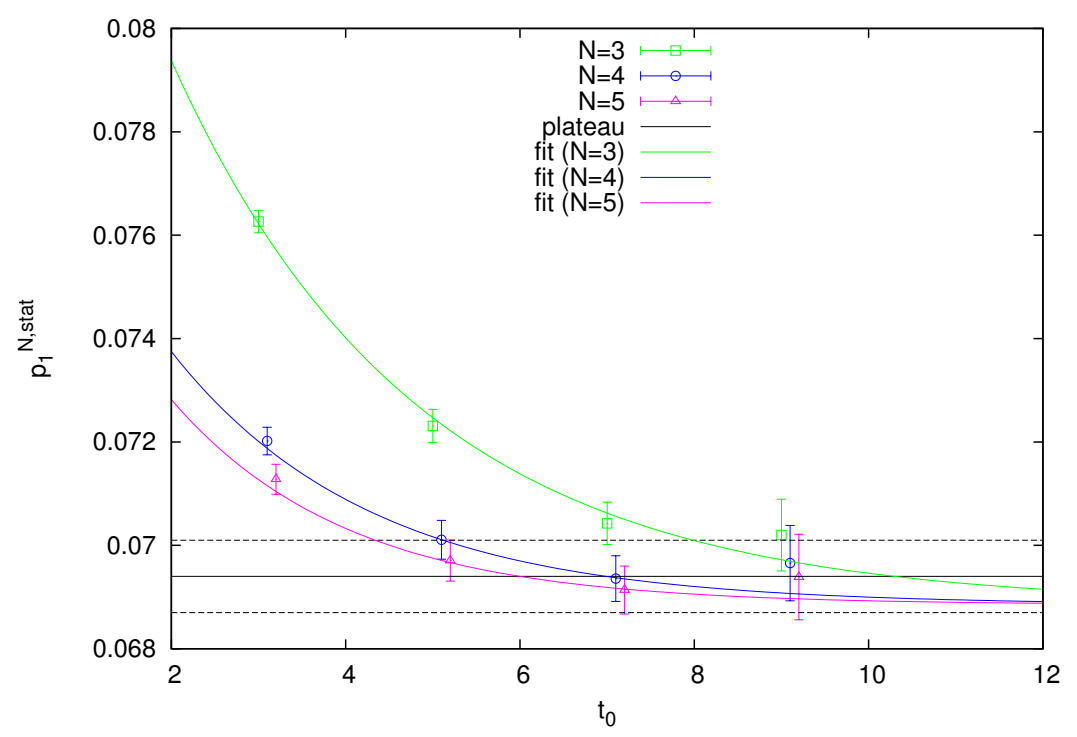

Figure 6. Plot of $p_{1}^{N \text {,stat }}\left(t_{0}+3 a, t_{0}\right)$ against $t_{0}$ in the same style as figure 2 .

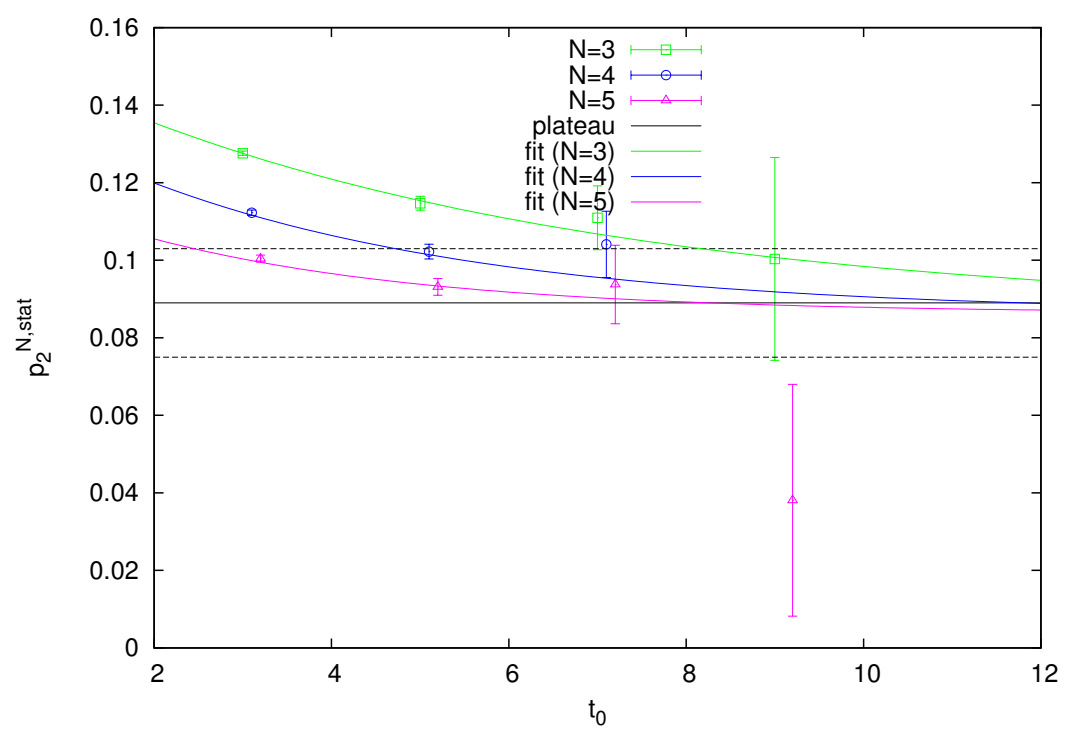

Figure 7. Plot of $p_{2}^{N \text {,stat }}\left(t_{0}+3 a, t_{0}\right)$ against $t_{0}$ in the same style as figure 2 .

the optimal plateau and its error bands. It is apparent not only that the data are very well described by the fitted functional forms, but also that the fits agree within errors with the more conservative plateau estimates.

To study how independent the results obtained from the GEVP are from the operator basis used, we have rerun our analysis for the static case using a different basis of operators where we exchanged some of the intermediate smearing levels against operators constructed by an additional application of the Laplacian. We found that the results for both the static energies and the static matrix elements were the same within errors as those obtained from our original basis. 


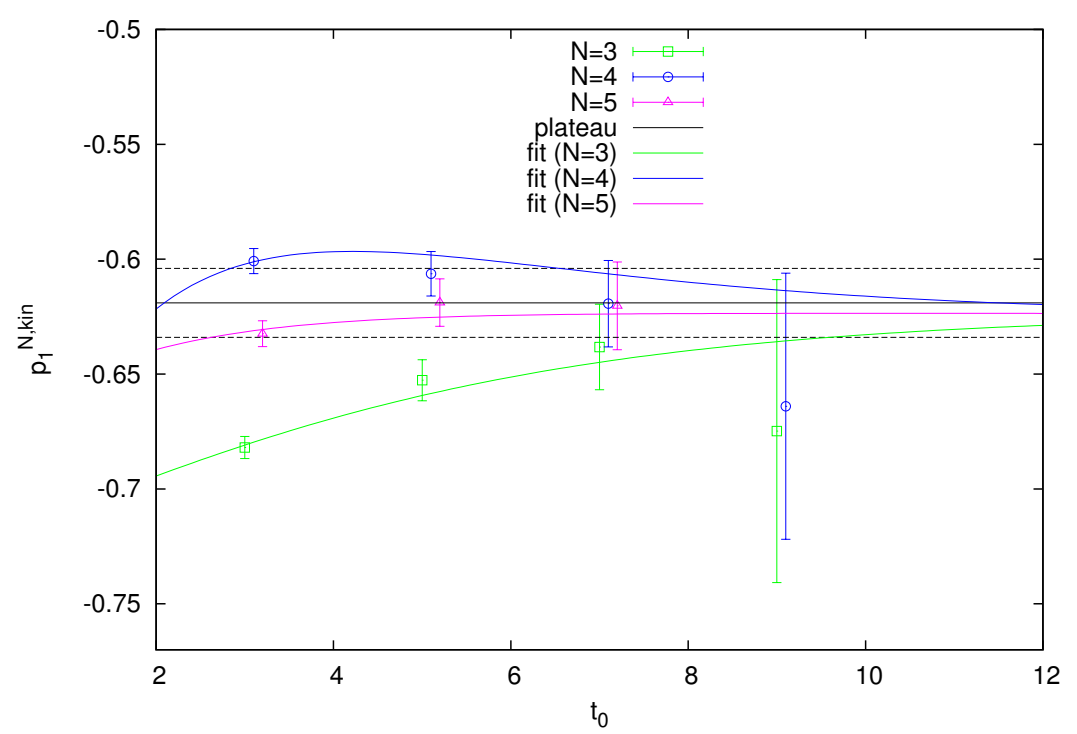

Figure 8. Plot of $p_{1}^{N \text {,kin }}\left(t_{0}+3 a, t_{0}\right)$ against $t_{0}$ in the same style as figure 2 .

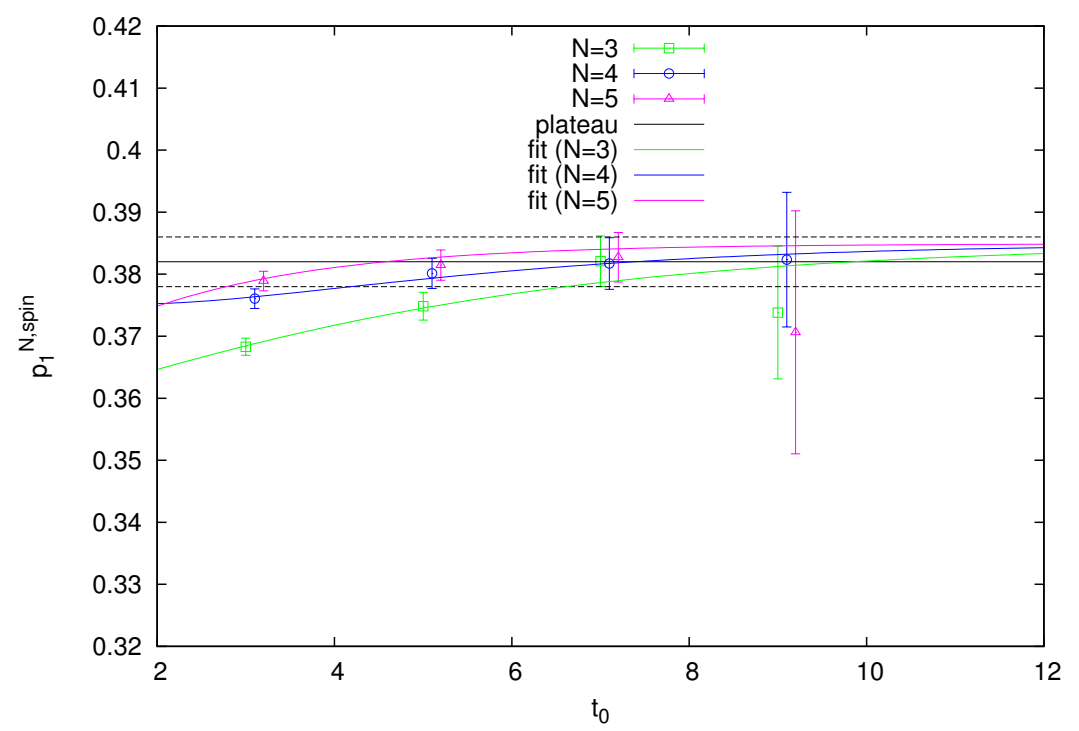

Figure 9. Plot of $p_{1}^{N \text {,spin }}\left(t_{0}+3 a, t_{0}\right)$ against $t_{0}$ in the same style as figure 2 .

\section{Discussion}

We have performed a theoretical analysis of the GEVP for lattice field theories, eq. (2.2). The $N \times N$ problem is expanded in terms of a convergent perturbative expansion around the unperturbed system defined by truncating the spectral representation of the correlation matrices after $N$ levels. The contribution of all the higher levels defines the perturbation.

The GEVP involves two time separations, $t_{0}, t$, where $t>t_{0}$. At large $t$ and fixed $t_{0}$, 


\begin{tabular}{|lllll|}
\hline Quantity & Full fit & Plateau fit & Plateau & Precision \\
\hline$a E_{1}^{\text {stat }}$ & $0.3026(4)$ & $0.3036(9)(2)$ & $5,9,12$ & $\lesssim 3 \mathrm{MeV}$ \\
$a E_{2}^{\text {stat }}$ & $0.483(3)$ & $0.484(7)(5)$ & $5,6,10$ & $\sim 20 \mathrm{MeV}$ \\
$a E_{3}^{\text {stat }}$ & $0.61(1)$ & $0.59(3)(2)$ & $5,6,11$ & $\sim 100 \mathrm{MeV}$ \\
$a E_{4}^{\text {stat }}$ & $0.66(1)$ & - & - & - \\
$a E_{5}^{\text {stat }}$ & $0.74(2)$ & - & - & - \\
$a E_{6}^{\text {stat }}$ & $0.79(4)$ & - & - & - \\
$a^{3 / 2} p_{1}^{\text {stat }}$ & $0.0689(2)$ & $0.0694(5)(2)$ & $5,8,12$ & $0.7 \%$ \\
$a^{3 / 2} p_{2}^{\text {stat }}$ & $0.086(3)$ & $0.089(10)(4)$ & $5,7,10$ & $12 \%$ \\
$a^{2} E_{1}^{\text {kin }}$ & $0.6731(4)$ & $0.6729(4)(1)$ & $3,5,6$ & $\lesssim 0.6 \mathrm{MeV}$ \\
$a^{2} E_{2}^{\text {kin }}$ & $0.691(5)$ & $0.69(2)(2)$ & $4,8,11$ & $\sim 40 \mathrm{MeV}$ \\
$a^{2} E_{1}^{\text {spin }}$ & $-0.0129(1)$ & $-0.0131(3)(1)$ & $4,8,9$ & $\lesssim 0.7 \mathrm{MeV}$ \\
$a^{2} E_{2}^{\text {spin }}$ & $-0.0106(4)$ & $-0.0113(9)(6)$ & $5,6,7$ & $\sim 2 \mathrm{MeV}$ \\
$a p_{1}^{\text {kin }}$ & $-0.622(8)$ & $-0.619(12)(3)$ & $5,5,8$ & $0.6 \%$ \\
$a p_{1}^{\text {spin }}$ & $0.385(2)$ & $0.382(3)(1)$ & $5,6,8$ & $0.7 \%$ \\
$a p_{1}^{\delta A}$ & $0.3120(3)$ & $0.315(3)(1)$ & $4,6,18$ & $0.1 \%$ \\
\hline
\end{tabular}

Table 1. The values of quantities of interest as determined from the fit and plateau picking procedure described in the text. The errors in the column headed "Plateau fit" are statistical and systematic. The column headed "Plateau" contains $N, t_{0}$ and $t_{\min }$ of the best plateau for energy levels, and $N, t_{0, \min }$ and $t$ of the best plateau for matrix elements. The column headed "Precision" contains an estimate of the contribution of the total error of that quantity to the total absolute error on the energy of the state, or to the total relative error of the decay constant, respectively. Note that terms such as $\omega_{\text {kin }} p_{1}^{\text {kin }}$ do not directly give the physical $1 / m$ correction since they contribute divergent contributions cancelled for example by $Z_{\mathrm{A}}^{\mathrm{HQET}}$.

the extracted energies $E_{n}^{\text {eff }}$ converge to the eigenvalues of the Hamiltonian with a rate

$$
\mathrm{O}\left(\mathrm{e}^{-\Delta E_{n} t}\right)
$$

where $\Delta E_{n}$ is the distance of $E_{n}$ to the closest energy level. Such a spectral gap is typically only a few hundred $\mathrm{MeV}$, requiring $t$ to be above $t>1 \mathrm{fm}$ for an appreciable suppression. One realizes, however, that the first order corrections are only of size

$$
\mathrm{O}\left(\mathrm{e}^{-\left(E_{N+1}-E_{n}\right) t}\right)
$$

where $E_{N+1}-E_{n}$ can be much bigger than $\Delta E_{n}$. At the second order in perturbation theory and beyond, the mixing of different levels introduces the slower decaying corrections, eq. (5.1) when $t_{0}$ is kept fixed, $t$ is taken large. We could show that the favorable suppression, eq. (5.2), is recovered to all orders if one chooses $t_{0} \geq t / 2$. This is possible because the mentioned mixing is suppressed exponentially in $t_{0}$. Thus, even if it is challenging numerically, a large $t_{0}$ (and $N$ ) is more important than a large $t$ to keep systematic errors small. In the numerical demonstration, section 4 , we have shown that this property can be used in practice. 
Furthermore, because of the exponential suppression of mixing with $t_{0}$, one can also write down interpolating fields, eq. (2.16), for all states with energies up to $E_{n}=E_{N}$. These fields are linear combinations of the $N$ fields one started from, with coefficients which depend on $t_{0}$. They are interpolating fields for the desired energy eigenstates up to corrections which decay as

$$
\mathrm{O}\left(\mathrm{e}^{-\left(E_{N+1}-E_{n}\right) t_{0}}\right)
$$

again with the large energy gap.

In a numerical example we could demonstrate eq. (5.2) and eq. (5.3) nicely by increasing $N, t_{0}, t$ starting from small values. The decrease of the correction terms is clearly observed, in particular in figure 2 and figure 6 , and the fit demonstrates that the derived formulae are (at least approximately) valid at the accessible values of $N, t_{0}, t$. This quantitative understanding of correction terms allows for a determination of energy levels and matrix elements with subdominant systematic errors.

In the considered HQET problem we achieved a sub-percent level (statistical + systematic) determination of the matrix elements needed for the B-meson decay constant, both at the leading and at the next-to-leading order in $1 / m_{\mathrm{b}}$. The ground state energy is obtained at the level of about $3 \mathrm{MeV}$. A more complete analysis at several values of $\beta$, including a continuum extrapolation, is currently in progress.

\section{Acknowledgments}

The authors are grateful to Ferenc Niedermayer and Peter Weisz for sharing their private note on the GEVP with us. We thank Nicolas Garron for useful discussions and an intense collaboration in the HQET project. This work is supported by the Deutsche Forschungsgemeinschaft in the SFB/TR 09, and by the European community through EU Contract No. MRTN-CT-2006-035482, "FLAVIAnet". T.M. also thanks the A. von Humboldt Foundation for support.

\section{A Recursive perturbative expansion}

\section{A.1 The general case}

Solving eq. (3.1) order by order in $\epsilon$ and considering the result at order $\epsilon^{k}$, the GEVP reads

$$
\begin{aligned}
0= & \left(A^{(0)}-\lambda_{n}^{(0)} B^{(0)}\right) v_{n}^{(k)}+\left(\Delta_{n}-\lambda_{n}^{(1)} B^{(0)}\right) v_{n}^{(k-1)} \\
& +\left(-\lambda_{n}^{(1)} B^{(1)}-\lambda_{n}^{(2)} B^{(0)}\right) v_{n}^{(k-2)}+\left(-\lambda_{n}^{(2)} B^{(1)}-\lambda_{n}^{(3)} B^{(0)}\right) v_{n}^{(k-3)} \\
& +\ldots+\left(-\lambda_{n}^{(k-1)} B^{(1)}-\lambda_{n}^{(k)} B^{(0)}\right) v_{n}^{(0)}
\end{aligned}
$$

where

Projecting with $v_{n}^{(0)}$ gives

$$
\Delta_{n}=A^{(1)}-\lambda_{n}^{(0)} B^{(1)} .
$$

$$
\lambda_{n}^{(k)} \rho_{n}=\left(v_{n}^{(0)}, \Delta_{n} v_{n}^{(k-1)}\right)-\sum_{l=1}^{k-1} \lambda_{n}^{(l)}\left(v_{n}^{(0)}, B^{(1)} v_{n}^{(k-1-l)}\right),
$$


and a projection with $v_{m}^{(0)}, m \neq n$ yields

$$
\begin{aligned}
& v_{n}^{(k)}=\sum_{m \neq n} \alpha_{m n}^{(k)} v_{m}^{(0)} \\
& \alpha_{m n}^{(k)} \rho_{m}=\left(v_{m}^{(0)}, B^{(0)} v_{n}^{(k)}\right) \\
& =\frac{1}{\lambda_{n}^{(0)}-\lambda_{m}^{(0)}}\left\{\left(v_{m}^{(0)}, \Delta_{n} v_{n}^{(k-1)}\right)-\sum_{l=1}^{k-1} \lambda_{n}^{(l)}\left(v_{m}^{(0)}, B^{(1)} v_{n}^{(k-1-l)}\right)\right. \\
& \left.-\sum_{l=1}^{k-1} \lambda_{n}^{(l)}\left(v_{m}^{(0)}, B^{(0)} v_{n}^{(k-l)}\right)\right\} .
\end{aligned}
$$

The combined recursions

$$
\alpha_{m n}^{(k)}=\rho_{m}^{-1} \frac{1}{\lambda_{n}^{(0)}-\lambda_{m}^{(0)}}\left\{\left(v_{m}^{(0)}, \Delta_{n} v_{n}^{(k-1)}\right)-\sum_{l=1}^{k-1} \lambda_{n}^{(l)}\left[\left(v_{m}^{(0)}, B^{(1)} v_{n}^{(k-1-l)}\right)+\rho_{m} \alpha_{m n}^{(k-l)}\right]\right\}
$$

and eq. (A.3) then determine the solution to arbitrary order in the perturbations starting from the initial values $\alpha_{m n}^{(0)}=\delta_{m n}, \lambda_{n}^{(0)}\left(t, t_{0}\right)=\mathrm{e}^{-E_{n}\left(t-t_{0}\right)}$.

\section{A.2 The case of Euclidean QFT}

We now apply this with

$$
\begin{aligned}
& A^{(0)}=C^{(0)}(t), \quad \epsilon A^{(1)}=C^{(1)}(t), \\
& B^{(0)}=C^{(0)}\left(t_{0}\right), \quad \epsilon B^{(1)}=C^{(1)}\left(t_{0}\right), \\
& v_{n}^{(0)}=u_{n} \quad \rightarrow \quad \rho_{n}=\mathrm{e}^{-E_{n} t_{0}},
\end{aligned}
$$

where we recall that

$$
C_{i j}^{(0)}(t)=\sum_{n=1}^{N} \mathrm{e}^{-E_{n} t} \psi_{n i} \psi_{n j}^{*}, \quad C_{i j}^{(1)}(t)=\sum_{n=N+1}^{\infty} \mathrm{e}^{-E_{n} t} \psi_{n i} \psi_{n j}^{*},
$$

and

$$
\psi_{n i}=\left\langle n\left|\hat{O}_{i}\right| 0\right\rangle, \quad \hat{H}|n\rangle=E_{n}|n\rangle .
$$

This means that $\epsilon v_{n}^{(1)}, \epsilon \lambda_{n}^{(1)} \ldots$ are corrections to the desired quantities which are the lowest order $u_{n}, \lambda_{n}^{(0)}$. We also recall the orthogonality

$$
\left(u_{n}, \psi_{m}\right)=\delta_{m n}, m, n \leq N .
$$

\section{A.3 Proof of eqs. (2.22) $-(2.24)$ to all orders}

The first step is to identify what exactly has to be shown concerning the behavior of $\lambda_{n}^{(k)}$ and $\alpha_{m n}^{(k)}$ to prove eqs. $(2.22)-(2.24)$. For $\varepsilon_{n}$ we expand

$$
\begin{aligned}
\varepsilon_{n}\left(t, t_{0}\right) & =-\partial_{t} \log \left(1+\sum_{k \geq 1} \epsilon^{k} \frac{\lambda_{n}^{(k)}\left(t, t_{0}\right)}{\lambda_{n}^{(0)}\left(t, t_{0}\right)}\right) \\
& =-\sum_{l=1}^{\infty} \frac{(-1)^{l+1}}{l} \sum_{k_{1}, \ldots, k_{l} \geq 1} \epsilon^{\sum_{i} k_{i}} \partial_{t}\left\{\frac{\lambda_{n}^{\left(k_{1}\right)}\left(t, t_{0}\right)}{\lambda_{n}^{(0)}\left(t, t_{0}\right)} \ldots \frac{\lambda_{n}^{\left(k_{l}\right)}\left(t, t_{0}\right)}{\lambda_{n}^{(0)}\left(t, t_{0}\right)}\right\} .
\end{aligned}
$$


If the conditions

$$
\partial_{t} \frac{\lambda_{n}^{(k)}\left(t, t_{0}\right)}{\lambda_{n}^{(0)}\left(t, t_{0}\right)}=\mathrm{O}\left(\mathrm{e}^{-\Delta E_{N+1, n} t}\right), \quad \frac{\lambda_{n}^{(k)}\left(t, t_{0}\right)}{\lambda_{n}^{(0)}\left(t, t_{0}\right)}=\mathrm{O}(1)
$$

are satisfied, one sees easily that each term in eq. (A.13) is of order $\mathrm{e}^{-\Delta E_{N+1, n} t}$. In other words eq. (A.14) is a sufficient condition for eq. (2.22) to hold. Next we expand

$$
\begin{aligned}
\left(\hat{\mathcal{A}}_{n}^{\mathrm{eff}}\right)^{\dagger}|0\rangle= & \mathrm{e}^{-\hat{H} t}\left(\hat{\mathcal{Q}}_{n}^{\mathrm{eff}}\right)^{\dagger}|0\rangle=\left[\mathrm{e}^{E_{n} t} \sum_{k \geq 0} \epsilon^{k} \sum_{n^{\prime} \geq 1} \mathrm{e}^{-E_{n^{\prime}} t}\left|n^{\prime}\right\rangle\left(\psi_{n^{\prime}}, v_{n}^{(k)}\left(t, t_{0}\right)\right)\right] \times\left[1+\varepsilon_{n}\right] \\
\times & {\left[1+\sum_{k_{1}, k_{2} \geq 1} \epsilon^{k 1+k 2} c_{k_{1}, k_{2}}\left(v_{n}^{\left(k_{1}\right)}\left(t, t_{0}\right), \mathrm{e}^{E_{n} t} C^{(0)}(t) v_{n}^{\left(k_{2}\right)}\left(t, t_{0}\right)\right)\right.} \\
& \left.+\sum_{k_{1}, k_{2} \geq 1} \epsilon^{k 1+k 2+1} \tilde{c}_{k_{1}, k_{2}}\left(v_{n}^{\left(k_{1}\right)}\left(t, t_{0}\right), \mathrm{e}^{E_{n} t} C^{(1)}(t) v_{n}^{\left(k_{2}\right)}\left(t, t_{0}\right)\right)\right]
\end{aligned}
$$

with some irrelevant coefficients $c_{k_{1}, k_{2}}, \tilde{c}_{k_{1}, k_{2}}$ and with $\varepsilon_{n}$ a shorthand for sums of terms $\varepsilon_{n}\left(t^{\prime}, t_{0}\right)$, which are all negligible provided eq. (2.22) holds. We express the various terms in eq. (A.15) through $\alpha_{m n}$ in order to arrive at conditions for these coefficients:

$$
\begin{aligned}
& {\left[\mathrm{e}^{E_{n} t} \sum_{k \geq 0} \sum_{n^{\prime} \geq 1} \mathrm{e}^{-E_{n^{\prime}} t}\left|n^{\prime}\right\rangle\left(\psi_{n^{\prime}}, v_{n}^{(k)}\left(t, t_{0}\right)\right)\right]} \\
& =\sum_{n^{\prime}=1}^{N} \sum_{m=1}^{N} \mathrm{e}^{-\left(E_{n^{\prime}}-E_{n}\right) t}\left|n^{\prime}\right\rangle \sum_{k \geq 0}\left(\psi_{n^{\prime}}, u_{m}\right) \alpha_{m n}^{(k)}\left(t, t_{0}\right)+\mathrm{O}\left(\mathrm{e}^{-\Delta E_{N+1, n} t}\right) \\
& =\sum_{m=1}^{N} \mathrm{e}^{-\left(E_{m}-E_{n}\right) t}|m\rangle \sum_{k \geq 0} \alpha_{m n}^{(k)}\left(t, t_{0}\right)+\mathrm{O}\left(\mathrm{e}^{-\Delta E_{N+1, n} t}\right) \\
& =|n\rangle+\sum_{m \neq n=1}^{N} \mathrm{e}^{-\left(E_{m}-E_{n}\right) t}|m\rangle \sum_{k \geq 1} \alpha_{m n}^{(k)}\left(t, t_{0}\right)+\mathrm{O}\left(\mathrm{e}^{-\Delta E_{N+1, n} t}\right) \\
& \left(v_{n}^{\left(k_{1}\right)}, \mathrm{e}^{E_{n} t} C^{(1)}(t) v_{n}^{\left(k_{2}\right)}\right)=\mathrm{O}\left(\mathrm{e}^{-\Delta E_{N+1, n} t}\right) \\
& \left(v_{n}^{\left(k_{1}\right)}, \mathrm{e}^{E_{n} t} C^{(0)}(t) v_{n}^{\left(k_{2}\right)}\right)=0 \quad \text { if } k_{1}=0 \text { or } k_{2}=0 \\
& \left(v_{n}^{\left(k_{1}\right)}, \mathrm{e}^{E_{n} t} C^{(0)}(t) v_{n}^{\left(k_{2}\right)}\right)=\sum_{m, m^{\prime}} \alpha_{m n}^{\left(k_{1}\right)}\left(t, t_{0}\right) \alpha_{m^{\prime} n}^{\left(k_{2}\right)}\left(t, t_{0}\right)\left(u_{m}, \mathrm{e}^{E_{n} t} C^{(0)}(t) u_{m}^{\prime}\right) \\
& =\sum_{m} \alpha_{m n}^{\left(k_{1}\right)}\left(t, t_{0}\right) \alpha_{m n}^{\left(k_{2}\right)}\left(t, t_{0}\right) \mathrm{e}^{-\left(E_{m}-E_{n}\right) t}
\end{aligned}
$$

A look at these terms shows that it is sufficient to prove

$$
\alpha_{m n}^{(k)}\left(t, t_{0}\right)= \begin{cases}\mathrm{O}\left(\mathrm{e}^{-\Delta E_{N+1, m} t_{0}}\right) & \text { for } m>n \\ \mathrm{O}\left(\mathrm{e}^{-\Delta E_{N+1, m} t_{0}} \mathrm{e}^{-\Delta E_{n, m}\left(t-t_{0}\right)}\right) & \text { for } m<n\end{cases}
$$




$$
\begin{aligned}
\partial_{t} \frac{\lambda_{n}^{(k)}\left(t, t_{0}\right)}{\lambda_{n}^{(0)}\left(t, t_{0}\right)} & =\mathrm{O}\left(\mathrm{e}^{-\Delta E_{N+1, n} t}\right) \\
\frac{\lambda_{n}^{(k)}\left(t, t_{0}\right)}{\lambda_{n}^{(0)}\left(t, t_{0}\right)} & =\mathrm{O}(1)
\end{aligned}
$$

for all $k$. (The last two conditions are sufficient to show that $\varepsilon_{n}\left(t, t_{0}\right)=\mathrm{O}\left(\mathrm{e}^{-\Delta E_{N+1, n} t}\right)$ ). Note that for $k=0$ the above conditions hold trivially.

As a next step we collect the large time behaviour of the different terms appearing in the recursions eq. (A.3) and eq. (A.6) for $\lambda^{(k)}, \alpha^{(k)}$ :

$$
\begin{aligned}
v_{n}^{(0)} & =\mathrm{O}(1), \\
\epsilon B^{(1)} & =\mathrm{O}\left(\mathrm{e}^{-E_{N+1} t_{0}}\right), \epsilon A^{(1)}=\mathrm{O}\left(\mathrm{e}^{-E_{N+1} t}\right), \\
\epsilon \Delta_{n} & =\mathrm{O}\left(\mathrm{e}^{-E_{N+1} t_{0}} \mathrm{e}^{-E_{n}\left(t-t_{0}\right)}\right)+\mathrm{O}\left(\mathrm{e}^{-E_{N+1} t}\right), \\
\epsilon \rho_{m}^{-1} B^{(1)} & =\mathrm{O}\left(\mathrm{e}^{-\left(E_{N+1}-E_{m}\right) t_{0}}\right), \\
\frac{\epsilon \rho_{m}^{-1} \Delta_{n}}{\lambda_{n}^{(0)}} & =\mathrm{O}\left(\mathrm{e}^{-\left(E_{N+1}-E_{m}\right) t_{0}}\right)+\mathrm{O}\left(\mathrm{e}^{-\left(E_{N+1}-E_{m}\right) t_{0}} \mathrm{e}^{-\left(E_{N+1}-E_{n}\right)\left(t-t_{0}\right)}\right),
\end{aligned}
$$

where in $\Delta_{n}$ we keep the two terms because the leading one has no $t$-dependence. For shortness we drop the $\mathrm{O}$ symbol from now on but just count orders and we use $t>t_{0}$. This means e.g. that $\mathrm{e}^{-E_{N+1} t_{0}}+\mathrm{e}^{-E_{n} t_{0}}=\mathrm{e}^{-E_{n} t_{0}}$. However, since derivatives with respect to $t$ are relevant we have to be careful not to drop $\mathrm{e}^{-E_{n} t}$ with respect to $\mathrm{e}^{-E_{n} t_{0}}$.

The shorthands

$$
\begin{aligned}
\eta_{N m}(t) & =\mathrm{O}\left(\mathrm{e}^{-\left(E_{N+1}-E_{m}\right) t}\right) \\
\gamma_{n m}(t) & =\frac{\mathrm{e}^{-E_{n} t}}{\mathrm{e}^{-E_{n} t}-\mathrm{e}^{-E_{m} t}} \\
& =\left\{\begin{array}{ll}
\sum_{j=0}^{\infty} \mathrm{e}^{-j\left(E_{m}-E_{n}\right) t} & \text { when } m>n \\
-\sum_{j=1}^{\infty} \mathrm{e}^{-j\left(E_{n}-E_{m}\right) t} & \text { when } n>m
\end{array},\right.
\end{aligned}
$$

are useful to discuss the large $t, t_{0}$ asymptotics,

$$
\begin{aligned}
\frac{\lambda_{n}^{(0)}}{\lambda_{n}^{(0)}-\lambda_{m}^{(0)}} & =\gamma_{n m}\left(t-t_{0}\right)=\left\{\begin{array}{ll}
1+\mathrm{O}\left(\mathrm{e}^{-\left(E_{m}-E_{n}\right) t}\right) & \text { when } m>n \\
\mathrm{O}\left(\mathrm{e}^{-\left(E_{n}-E_{m}\right) t}\right) & \text { when } n>m
\end{array},\right. \\
\frac{\rho_{m^{\prime}}^{-1} \epsilon \Delta_{n}}{\lambda_{n}^{(0)}-\lambda_{m}^{(0)}} & =\eta_{N m^{\prime}}\left(t_{0}\right)\left[1+\eta_{N n}\left(t-t_{0}\right)\right] \gamma_{n m}\left(t-t_{0}\right) \\
\frac{\rho_{m^{\prime}}^{-1} \epsilon B^{(1)} \lambda_{n}^{(0)}}{\lambda_{n}^{(0)}-\lambda_{m}^{(0)}} & =\eta_{N m^{\prime}}\left(t_{0}\right) \gamma_{n m}\left(t-t_{0}\right) .
\end{aligned}
$$

In this notation the asymptotics of the first order corrections are

$$
\begin{aligned}
\frac{\epsilon \lambda_{n}^{(1)}}{\lambda_{n}^{(0)}} & =\eta_{N n}\left(t_{0}\right)\left[1+\eta_{N n}\left(t-t_{0}\right)\right], \\
\epsilon \alpha_{m n}^{(1)} & =\eta_{N m}\left(t_{0}\right)\left[1+\eta_{N n}\left(t-t_{0}\right)\right] \gamma_{n m}\left(t-t_{0}\right),
\end{aligned}
$$


while the recursions are

$$
\begin{aligned}
\epsilon^{k} \frac{\lambda_{n}^{(k)}}{\lambda_{n}^{(0)}}= & \epsilon^{k} \frac{\lambda_{n}^{(1)}}{\lambda_{n}^{(0)}} \alpha^{(k-1)}+\epsilon^{k-1} \sum_{l=1}^{k-1} \frac{\lambda_{n}^{(l)}}{\lambda_{n}^{(0)}} \eta_{N n}\left(t_{0}\right) \alpha^{(k-1-l)} \\
= & \frac{\lambda_{n}^{(1)}}{\lambda_{n}^{(0)}}\left\{\epsilon^{k} \alpha^{(k-1)}+\epsilon^{k-1} \sum_{l=1}^{k-1} \frac{\lambda_{n}^{(l)}}{\lambda_{n}^{(1)}} \eta_{N n}\left(t_{0}\right) \alpha^{(k-1-l)}\right\} \\
\epsilon^{k} \alpha_{m n}^{(k)}= & \epsilon^{k} \alpha_{m n}^{(1)} \alpha^{(k-1)}+\epsilon^{k-1} \sum_{l=1}^{k-1} \frac{\lambda_{n}^{(l)}}{\lambda_{n}^{(0)}} \eta_{N m}\left(t_{0}\right) \alpha^{(k-1-l)} \gamma_{n m}\left(t-t_{0}\right) \\
& +\epsilon^{k} \sum_{l=1}^{k-1} \frac{\lambda_{n}^{(l)}}{\lambda_{n}^{(0)}} \alpha_{m n}^{(k-l)} \gamma_{n m}\left(t-t_{0}\right) \\
= & \alpha_{m n}^{(1)}\left\{\epsilon^{k} \alpha^{(k-1)}+\sum_{l=1}^{k-1} \frac{\lambda_{n}^{(l)}}{\lambda_{n}^{(1)}}\left[\epsilon^{k-1} \alpha^{(k-1-l)} \eta_{N n}\left(t_{0}\right)+\epsilon^{k} \alpha_{m n}^{(k-l)}\right]\right\}
\end{aligned}
$$

where $\alpha^{(k)}=\max _{m} \alpha_{m n}^{(k)}$. We finally will need the start values of the derivatives

$$
\begin{aligned}
\epsilon \partial_{t} \frac{\lambda_{n}^{(1)}}{\lambda_{n}^{(0)}}= & \eta_{N n}\left(t_{0}\right) \eta_{N n}\left(t-t_{0}\right)=\eta_{N n}(t) \\
\epsilon \partial_{t} \alpha_{m n}^{(1)}= & \eta_{N m}\left(t_{0}\right)\left[\partial_{t} \gamma_{n m}\left(t-t_{0}\right)+\partial_{t} \eta_{N n}\left(t-t_{0}\right) \gamma_{n m}\left(t-t_{0}\right)\right. \\
& \left.\quad+\eta_{N n}\left(t-t_{0}\right) \partial_{t} \gamma_{n m}\left(t-t_{0}\right)\right] \\
= & \eta_{N m}\left(t_{0}\right) \mathrm{e}^{-\left|E_{m}-E_{n}\right|\left(t-t_{0}\right)}
\end{aligned}
$$

where eq. (A.31) has been used. In particular the relation

$$
\epsilon \eta_{N n}\left(t_{0}\right) \partial_{t} \alpha_{m n}^{(1)} \leq \eta_{N n}(t) \quad \text { if } \quad t_{0} \geq t / 2
$$

or equivalently

$$
\epsilon \partial_{t} \alpha_{m n}^{(1)} \leq \eta_{N n}\left(t-t_{0}\right) \quad \text { if } \quad t_{0} \geq t / 2
$$

will be relevant.

In terms of the abreviations

$$
r_{k}=\epsilon^{k-1} \frac{\lambda_{n}^{(k)}}{\lambda_{n}^{(1)}}, \quad x_{k}=\epsilon^{k} \alpha_{m n}^{(k)}, \quad X_{k}=\epsilon^{k} \alpha^{(k)}=\epsilon^{k} \max _{m} \alpha_{m n}^{(k)}
$$

we want to show that

$$
\begin{aligned}
x_{k} & =\eta_{N m}\left(t_{0}\right) \gamma_{n m}\left(t-t_{0}\right), & r_{k} & =\mathrm{O}(1), \\
\partial_{t} x_{k} & =\eta_{N n}\left(t-t_{0}\right), & \partial_{t} r_{k} & =\eta_{N n}\left(t-t_{0}\right),
\end{aligned}
$$

which immediately implies eq. (A.20) to eq. (A.22) and thus proves eq. (2.22) to eq. (2.24). For $k=1$, eq. (A.43) and eq. (A.44) are satisfied by the start values derived above. The induction steps from $k-1$ to $k$ for $k \geq 2$ are very simple (remember we assume $t_{0} \geq t / 2$ ):

$$
r_{k}=X_{k-1}+\eta_{N n}\left(t_{0}\right) \sum_{l=1}^{k-1} r_{l} X_{k-1-l}=\max _{m} \eta_{N m}\left(t_{0}\right) \gamma_{n m}\left(t-t_{0}\right)=\mathrm{O}(1),
$$




$$
\begin{gathered}
x_{k}=x_{1}\left\{r_{k}+\sum_{l=1}^{k-1} r_{l} x_{k-l}\right\}=\eta_{N m}\left(t_{0}\right) \gamma_{n m}\left(t-t_{0}\right), \\
\partial_{t} r_{k}=\partial_{t} X_{k-1}+\eta_{N n}\left(t_{0}\right) \sum_{l=1}^{k-1} \partial_{t}\left[r_{l} X_{k-1-l}\right]=\eta_{N n}\left(t-t_{0}\right), \\
\partial_{t} x_{k}=\partial_{t}\left[x_{1} r_{k}\right]+\sum_{l=1}^{k-1} \partial_{t}\left[x_{1} r_{l} x_{k-l}\right]=\eta_{N n}\left(t-t_{0}\right) .
\end{gathered}
$$

They conclude our proof.

\section{References}

[1] K.G. Wilson, talk at the Abingdon Meeting on Lattice Gauge Theories, (1981).

[2] B. Berg, Glueball calculations in lattice gauge theories, in Paris 1982, Proceedings, High Energy Physics, pg. 272 [SPIRES].

[3] C. Michael and I. Teasdale, Extracting glueball masses from lattice QCD, Nucl. Phys. B 215 (1983) 433 [SPIRES].

[4] C. Michael, Adjoint sources in lattice gauge theory, Nucl. Phys. B 259 (1985) 58 [SPIRES].

[5] N.A. Campbell, L.A. Griffiths, C. Michael and P.E.L. Rakow, Mesons with excited glue from SU(3) lattice gauge theory, Phys. Lett. B 142 (1984) 291 [SPIRES].

[6] T. DeGrand and C.E. DeTar, Lattice methods for quantum chromodynamics, World Scientific, New Jersey U.S.A. (2006).

[7] A.O Cais, D. Leinweber, S. Mahbub and T. Williams, A fitting robot for variational analysis, arXiv:0812.1872 [SPIRES].

[8] Hadron Spectrum collaboration, J.M. Bulava et al., Excited nucleon spectrum with two flavors of dynamical fermions, arXiv:0810.0253 [SPIRES].

[9] S. Prelovsek and D. Mohler, A lattice study of light scalar tetraquarks, Phys. Rev. D 79 (2009) 014503 [arXiv: 0810.1759] [SPIRES].

[10] T. Burch, C. Hagen, C.B. Lang, M. Limmer and A. Schafer, Excitations of single-beauty hadrons, Phys. Rev. D 79 (2009) 014504 [arXiv: 0809.1103] [SPIRES].

[11] C. Gattringer et al., Hadron spectroscopy with dynamical chirally improved fermions, arXiv:0812.1681 [SPIRES].

[12] G.S. Bali and F. Bursa, Mesons at large $N_{c}$ from lattice QCD, JHEP 09 (2008) 110 [arXiv:0806.2278] [SPIRES].

[13] C. Gattringer, L.Y. Glozman, C.B. Lang, D. Mohler and S. Prelovsek, Derivative sources in lattice spectroscopy of excited mesons, Phys. Rev. D 78 (2008) 034501 [arXiv:0802.2020] [SPIRES].

[14] C. Ehmann and G. Bali, Charmonium spectrum including higher spin and exotic states, PoS (LATTICE 2007) 094 [arXiv: 0710.0256] [SPIRES].

[15] J. Danzer and C. Gattringer, Excited state spectroscopy in the lattice Gross-Neveu model, PoS (LATTICE 2007) 092 [arXiv:0710.1711] [SPIRES]. 
[16] S. Basak et al., Lattice QCD determination of patterns of excited baryon states, Phys. Rev. D 76 (2007) 074504 [arXiv:0709.0008] [SPIRES].

[17] J.J. Dudek, R.G. Edwards, N. Mathur and D.G. Richards, Charmonium excited state spectrum in lattice QCD, Phys. Rev. D 77 (2008) 034501 [arXiv:0707.4162] [SPIRES].

[18] J. Foley, A. O'Cais, M. Peardon and S.M. Ryan, Radial and orbital excitations of static-light mesons, Phys. Rev. D 75 (2007) 094503 [hep-lat/0702010] [SPIRES].

[19] T. Burch and C. Ehmann, Couplings of hybrid operators to ground and excited states of bottomonia, Nucl. Phys. A 797 (2007) 33 [hep-lat/0701001] [SPIRES].

[20] M. Lüscher and U. Wolff, How to calculate the elastic scattering matrix in two-dimensional quantum field theories by numerical simulation, Nucl. Phys. B 339 (1990) 222 [SPIRES].

[21] B. Blossier, G. von Hippel, T. Mendes, R. Sommer and M. Della Morte, Efficient use of the generalized eigenvalue problem, PoS (LATTICE 2008) 135 [arXiv:0808.1017] [SPIRES].

[22] M. Lüscher, Construction of a selfadjoint, strictly positive transfer matrix for euclidean lattice gauge theories, Commun. Math. Phys. 54 (1977) 283 [SPIRES].

[23] M. Creutz, Gauge fixing, the transfer matrix and confinement on a lattice, Phys. Rev. D 15 (1977) 1128 [SPIRES].

[24] M. Lüscher and P. Weisz, Definition and general properties of the transfer matrix in continuum limit improved lattice gauge theories, Nucl. Phys. B 240 (1984) 349 [SPIRES].

[25] S. Necco, Universality and scaling behavior of $R G$ gauge actions, Nucl. Phys. B 683 (2004) 137 [hep-lat/0309017] [SPIRES].

[26] N. Irges and F. Knechtli, Lattice gauge theory approach to spontaneous symmetry breaking from an extra dimension, Nucl. Phys. B 775 (2007) 283 [hep-lat/0609045] [SPIRES].

[27] ALPHA collaboration, F. Knechtli and R. Sommer, String breaking in SU(2) gauge theory with scalar matter fields, Phys. Lett. B 440 (1998) 345 [hep-lat/9807022] [SPIRES].

[28] ALPHA collaboration, F. Knechtli and R. Sommer, String breaking as a mixing phenomenon in the SU(2) Higgs model, Nucl. Phys. B 590 (2000) 309 [hep-lat/0005021] [SPIRES].

[29] O. Philipsen and H. Wittig, String breaking in non-Abelian gauge theories with fundamental matter fields, Phys. Rev. Lett. 81 (1998) 4056 [Erratum ibid. 83 (1999) 2684] [hep-lat/9807020] [SPIRES].

[30] SESAM collaboration, G.S. Bali, H. Neff, T. Duessel, T. Lippert and K. Schilling, Observation of string breaking in QCD, Phys. Rev. D 71 (2005) 114513 [hep-lat/0505012] [SPIRES].

[31] M. Pepe and U.J. Wiese, From decay to complete breaking: pulling the strings in $\mathrm{SU}(2)$ Yang-Mills theory, arXiv:0901.2510 [SPIRES].

[32] F. Niedermayer and P. Weisz, unpublished notes (1998).

[33] A. Hasenfratz and F. Knechtli, Flavor symmetry and the static potential with hypercubic blocking, Phys. Rev. D 64 (2001) 034504 [hep-lat/0103029] [SPIRES].

[34] A. Hasenfratz, R. Hoffmann and F. Knechtli, The static potential with hypercubic blocking, Nucl. Phys. Proc. Suppl. 106 (2002) 418 [hep-lat/0110168] [SPIRES].

[35] M. Della Morte, A. Shindler and R. Sommer, On lattice actions for static quarks, JHEP 08 (2005) 051 [hep-lat/0506008] [SPIRES]. 
[36] B. Sheikholeslami and R. Wohlert, Improved continuum limit lattice action for QCD with Wilson fermions, Nucl. Phys. B 259 (1985) 572 [SPIRES].

[37] M. Lüscher, S. Sint, R. Sommer, P. Weisz and U. Wolff, Non-perturbative O(a) improvement of lattice QCD, Nucl. Phys. B 491 (1997) 323 [hep-lat/9609035] [SPIRES].

[38] J. Foley et al., Practical all-to-all propagators for lattice QCD, Comput. Phys. Commun. 172 (2005) 145 [hep-lat/0505023] [SPIRES].

[39] B. Blossier et al., in preparation.

[40] S. Gusken et al., Nonsinglet axial vector couplings of the baryon octet in lattice QCD, Phys. Lett. B 227 (1989) 266 [SPIRES].

[41] APE collaboration, M. Albanese et al., Glueball masses and string tension in lattice QCD, Phys. Lett. B 192 (1987) 163 [SPIRES].

[42] S. Basak et al., Combining quark and link smearing to improve extended baryon operators, PoS (LAT2005) 076 [hep-lat/0509179] [SPIRES].

[43] M. Della Morte, N. Garron, M. Papinutto and R. Sommer, Heavy quark effective theory computation of the mass of the bottom quark, JHEP 01 (2007) 007 [hep-ph/0609294] [SPIRES].

[44] B. Blossier, M. Della Morte, N. Garron and R. Sommer, Heavy-light decay constant at the $1 / m$ order of HQET, PoS (LATTICE 2007) 245 [arXiv:0710.1553] [SPIRES].

[45] F. Niedermayer, P. Rufenacht and U. Wenger, Fixed point gauge actions with fat links: scaling and glueballs, Nucl. Phys. B 597 (2001) 413 [hep-lat/0007007] [SPIRES]. 